九州大学学術情報リポジトリ

Kyushu University Institutional Repository

Redescription of Cryptopecten yanagawaens is (NOMURA and ZINBO) : Paleontological Study of the Molluscan Fauna from the Moniwa Formation Part-2

Sato, Yoshio

Faculty of Science, Kyushu University

https://doi.org/10.5109/1546098

出版情報：九州大學理學部紀要：Series D, Geology. 25 (2)，pp.247-269，1984-11-15. Faculty of Science, Kyushu University バージョン :

権利関係 : 
Mem. Fac. Sci., Kyushu Univ., Ser. D, Geol., Vol. XXV, No. 2, pp. 247-269, text-figs. 1-7, tables 1-2, plates 36-37, Nobember 15, 1984

\title{
Redescription of Cryptopecten yanagawaensis (NOMURA and ZINBo) (Paleontological Study of the Molluscan Fauna from the Moniwa Formation Part-2)
}

\author{
Yoshio SATO
}

\begin{abstract}
This paper gives a redescription of a Miocene pectinid, Cryptopecten yanagawaensis (NOMURA and ZINBO) which occurs mainly in the beds aged $16 \mathrm{Ma}$, the early Medial Miocene. The present species is one of the most characteristic ones of the molluscan fauna of the Moniwa Formation and is presumed to be ancestor of the Pliocene and Recent species, C. vesiculosus (DUNKER). By way of block sampling method the writer collected more than 200 free specimens from 18 samples taken from the Moniwa Formation which yields many best preserved specimens. Redescription of the species is made by applying the results from paleoautoecological analysis. This paper also refers to the geographic and stratigraphic distribution and paleoecology of the species.
\end{abstract}

\section{Introduction}

The present article is the second report on the molluscan fauna of the Moniwa Formation distributed in the environs of Sendai, Miyagi Prefecture, Northeast Japan.

It is well known that the Moniwa Formation yields various kinds of megafossils such as molluscs, brachiopods, barnacles, corals, echinoids and vertebratebones in association with micro-fossils such as foraminifers and ostracods. These rich faunas have been studied by many specialists. Subsequently, the molluscan faunas have been restudied with proposal of some new taxa (HATAI, MASUdA and NodA, 1973 and SATo, 1982).

Sedimentary environments of the Moniwa Formation were summarized by OYAMA (1954) and MASUDA (1969), Who presumed a marine condition influenced by warm water of open sea.

Recently, a systematic study of the Moniwa fauna was carried out, in which many block samples were collected from each fossil locality of the Moniwa Formation and treated in the water bath in order to recognize the whole paleontological feature of fossil assemblages and to get environmental information (SAT0, 1979 and 1982). A number of species that had not been reported before were found by this procedure. NomURA's pioneer work (1940) is very valuable but it is taxonomically insufficient, and nothing has been done in paleosynecology. Because his descriptions were based on the specimens obtained in an 
unsystematic manner and lack comparison with specimens from other Tertiary deposits and also those with Recent species. Furthermore, he didn't treat the species on the basis of the population concept. Such being the case the writer has been working on revision and description of these molluscs in view of the modern taxonomy.

The shell height, width, depth, apical angle, length of both ears, and number of radial ribs are measured, and the ontogenetical change of the apical angle, proportion of shell height to width, number of radial ribs and differentiation of radial ribs are examined in growth series.

MORISHITA's $R \delta$, coefficient of interspecific association among the characteristic species, is calculated. Cryptopecten yanagawaensis shows harmonic association with Glycymeris derelicta (YoKoYAMA) and Placopecten nomurai MASUDA. Present species is a typical member of Placopecten nomurai MASUDA C. yanagawaensis (NOMURA and ZINBO) community.

\section{Acknowledgements}

The writer takes this opportunity to express his deepest gratitude to Professor Tsugio Shuto of the Department of geology, Faculty of Science, Kyushu University, for his supervision during the whole course of the present study and critically reading the manuscript, and wishes to dedicate this article to this memorial volume in honour of Prof. SHUTO.

Acknowledgements are due to Professor Koichiro MASUdA of the Miyagi University of Education for his valuable suggestion on various ways and photographic works.

During the course of this study the writer has received support from the following persons to whome he takaes this opportunity to express his deep gratitude; Professor Itaru HAYAMI, department of geology, Faculty of Science of the University of Tokyo, for his information of recent species of Cryptopecten, Dr. Shoichi Shimoyama, Department of Geology, Kyushu University, for his discussion on paleoautoecology, Dr. Kenshiro OGASAWARA of the Institute of Geology and Paleontology, Tohoku University, for his permission to study the specimens preserved there.

\section{Stratigraphical setting of the Moniwa Formation}

The Moniwa Formation, distributed in the southwestern area of Sendai City, is a constituent of the Natori Group. The latter is composed of the Tsukinoki, Takadate, Moniwa, Hatatate and Tsunaki foramtion in ascending order and represents a standard section of the Miocene deposits in the Pacific coast region of Northeast Japan (Hanzawa, S., HataI, K., IWAI, J. Kitamura, N. and Shibata, T., 1953).

The Moniwa Formation covers the Takadate Formation unconformably. Its basal conglomerate was widely traced by SATo (1979). The Hatatate Formation conformably covers the Moniwa Formation and the boundary between 
them is defined by a lapilli tuff at the east end of the type section of the Moniwa Formation. In the southwestern area, the transitional part of lithology from green coarse grained sandstone to white fine grained sandstone or silt is considered to correspond to the boundary mentioned above. While the Moniwa Formation is nearly flat in most area, it shows a gentle northward dip with the strike of E-W trend in the eastern area. The Moniwa Formation is distributed surrounding the lower Takadate Formation and shows steep dip at some places where it abuts on the Takadate Formation or is intruded by intrusive rocks. Thickness of the Moniwa Formation ranges from 3 meters to 28 meters and becomes thinner southward from its type locality. Geological age of the Moniwa Formation was assigned to the Early Miocene by molluscan fossils by MASUDA (1973 a). Subsequently, ODA and SAKAI (1977) recognized Globigerinoides sicanus/Globigerinatella insueta Zone in the Moniwa Formation and referred it to N 8, the Early Miocene. Recently, Tsuchi (1984) and TsuchI and SHUTO (1984) also considered that this formation corresponds to belong to $\mathrm{N}$ $8 \mathrm{~b}$, early Medial Miocene.

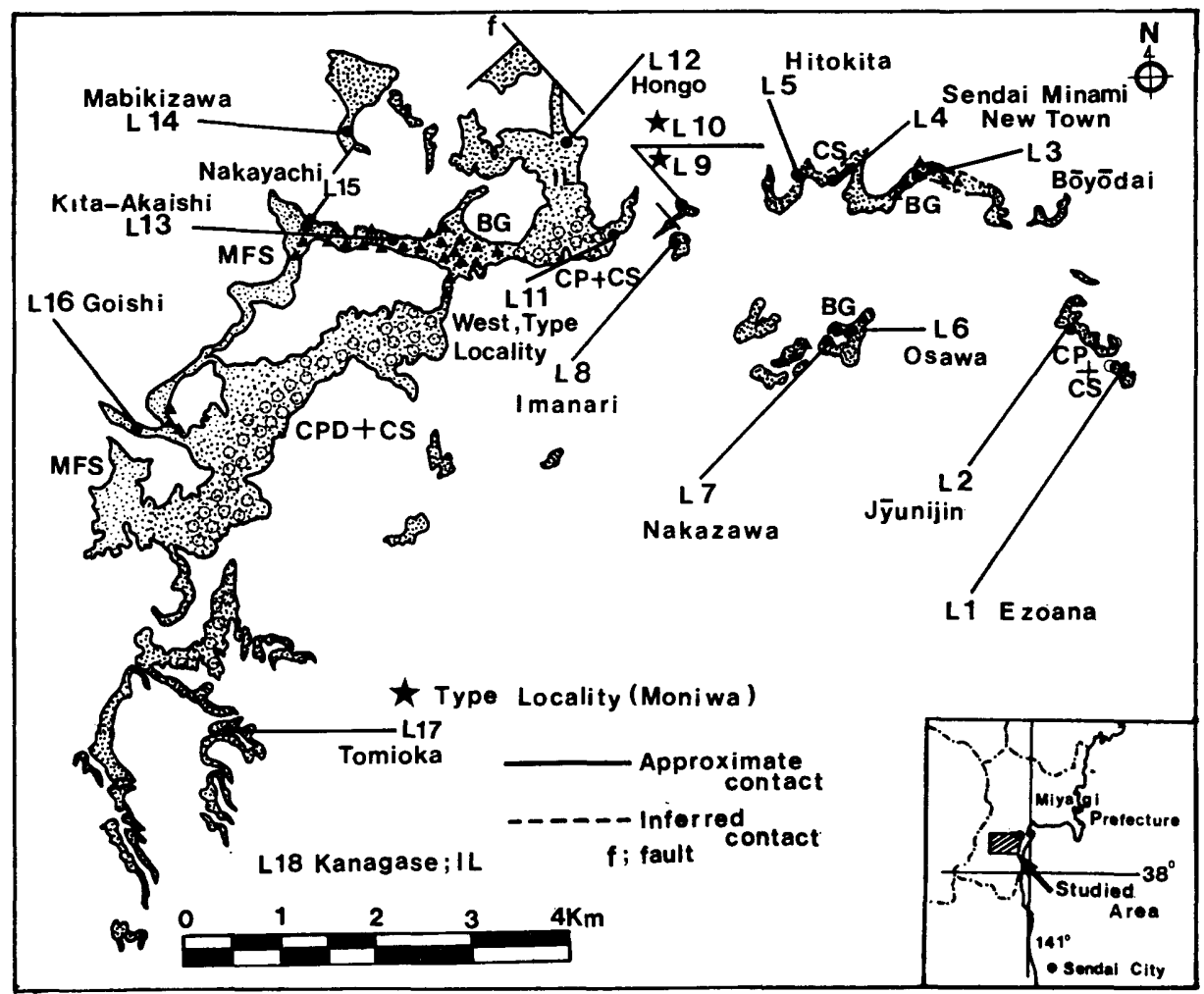

Fig. 1. Horizontal distribution of rock facies and fossil localities. CS (Coarse grained sandstone); MFS (Medium to fine grained standstone); IL (Impure limestone); CPD + CS (Dioritic pebble bearing conglomeratic coarse grained sandstone); CP+CS (Pebble bearing conglameratic coarse grained sandstone). L 18 (Kanagase) is located $14 \mathrm{~km}$ south from L 17 . 
Distribution and lithology of the Moniwa Formation is shown in Fig. 1. This foramtion is generally composed of coarse grained sandstone with andesite pebbles and granules and it gradually grades upward into conglomeratic coarse grained sandstone to coarse grained sandstone. In eastern area greenish coarse grained sandstone is predominant, while blush medium grained sandstone is found in the western area. Sediments are not so hard that fossils are easily detached from the block samples except for the calcareous coarse and fine grained sandstone at fossil locality L 9 and L 13. Pebbles and granules in the Moniwa Formation are so thin and flat that the formation is easily discriminated from other formations. Lithology of these pebbles and granules changes from andesitic to dioritic southward. Surface of the boulders is very smooth and occasionally bored by boring shells (MASUDA, 1968). Calcareous tubes made by worms and attachment scar of Balanus and Ostrea sp. are observed on the surface of boulders at L $3, \mathrm{~L} 8$ and L 13 .

\section{Fossil Localities and Occurrence of Fossils}

Eighteen fossil localities are studied (Fig. 1). L 9 and L 10 are situated along the type section of the Moniwa Formation. Stratigraphic position of $\mathrm{L} 10$ is five meters above L 9. L 13 at Kita-akaishi is the same locality as that of Nomura (1940). Precise description of fossil localities of the present species are listed in the separate chapter.

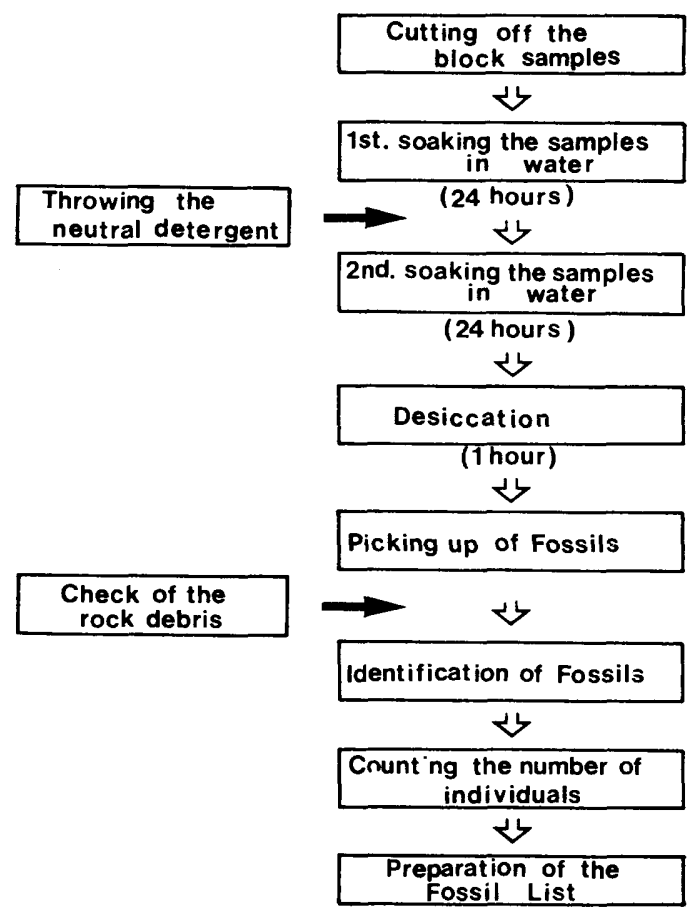

Fig. 3. Treatment of block samples. 
Fossils mainly consist of bivalves. They are generally accumulated and inarticulated in fossil beds. Mode of occurrence can be classified into the following three types: (1) shells are accumulated and orientated nearly parallel to the bedding plane, with their convex side upwards, (2) shells accumulated and orientated obliquely or perpendicularly to the bedding palne with their convex side turned dowunward, (3) shells trapped and accumulated in boulder gravels. Of them the type (2) predominates over others.

\section{Method of Sampling and Treatment of Black Samples}

Sampling localities where shells are expected to distribute uniformly in the shell bed are carefully selected. The shell bed is cut perpendicularly to the bedding plane at each sampling locality to obtain a block sample of $30 \mathrm{~cm}$ long, $30 \mathrm{~cm}$ wide and $20 \mathrm{~cm}$ thick. Number of blocks at one locality depends upon density of shells in the matrix. Sampling procedure is continued successively until total number of shells exceeds three hundreds. At L 2, additional samples were obtained in order to get more than two hundred valves of the present species. Treatment of block samples is shown in Fig. 2. They are soaked in water for two days and fossils are detached from matrix one hour after leaving water. A fractured shell is counted as an individual, if it holds twothirds or more of the entire shell is preserved and also the measurement of shell height is possible.

\section{Characteristic Species of the Moniwa Formation}

Characteristic species of megafossils such as molluscs, brachiopods and simple corals are listed in Fig. 5. Species occurring at more than six localities with more than fifty individuals are defined in this paper as characteristic species. Characteristic species all indicate a warm, shallow to neritic and open marine environment in which they lived. Pectinidae is by far predominant over the others and the present species is ranked at ninth in the characteristic species. It can be considered that molluscan assemblages of the Moniwa Formation are shallower subtidal ones on the sand, sand with pebbles and rocky bottom belonging to the Kadonosawa Fauna (CHINZEI, 1983) among the Neogene Tertiary molluscan faunas.

\section{Description of Cryptopecten yanagawaensis (NOMURA and ZINBO)}

Genus Cryptopecten DALL, BARTSCH \& REHDER, 1938

Type-species:-Cryptopecten alli DALL, BARTSCH \& REHDER

by orginal designation

Cat. No. 173194, 190440 and 335667. (United States National Museum) No. 173194 Measurements on ; Height $22.1 \mathrm{~mm}$, length, $22.8 \mathrm{~mm}$, diameter of single valve, $3.3 \mathrm{~mm}$. 
Table 1. Measurements of Cryptopecten yanagawaensis (NomURA and ZINBO). Right valve.

\begin{tabular}{|c|c|c|c|c|c|c|c|c|c|c|}
\hline Reg. No. & Loc. & $\mathrm{H}$ & W & $\mathrm{E}_{1}$ & $\mathbf{E}_{2}$ & $\mathbf{D}$ & $\mathrm{C}$ & A.A. & $\mathrm{H} / \mathrm{W}$ & N.R. \\
\hline GK-L9294 & $\mathrm{L} 2$ & 10.35 & 10.80 & 4.10 & 2.60 & 2.40 & 0.70 & $93^{\circ}$ & 0.96 & 21 \\
\hline 9295 & L2 & 11.00 & 11.20 & 4.10 & 2.90 & 1.95 & 0.45 & $91^{\circ}$ & 0.98 & 23 \\
\hline 9296 & L2 & 12.10 & 12.10 & 4.10 & 3.30 & 2.50 & 0.65 & $93^{\circ}$ & 1.00 & 22 \\
\hline 9297 & L18 & 12.60 & 11.70 & 3.40 & 3.20 & 2.50 & 0.55 & $89^{\circ}$ & 1.08 & 21 \\
\hline 9298 & L2 & 12.80 & 12.40 & 4.10 & 3.40 & 2.80 & + & $90^{\circ}$ & 1.03 & 22 \\
\hline 9299 & L2 & 14.50 & 14.20 & 4.80 & 3.90 & 3.20 & 0.80 & $90^{\circ}$ & 1.02 & 24 \\
\hline 9300 & L2 & 14.50 & 14.80 & 4.70 & 3.60 & 3.65 & 0.85 & $91^{\circ}$ & 0.98 & 23 \\
\hline 9301 & L2 & 14.50 & 13.60 & 4.50 & 2.50 & 2.65 & 0.65 & $91^{\circ}$ & 1.07 & 23 \\
\hline 9302 & L2 & 14.70 & 13.80 & 4.75 & 3.30 & 3.70 & 0.85 & $90^{\circ}$ & 1.07 & 21 \\
\hline 9303 & L2 & 14.70 & 14.30 & 5.35 & 3.60 & 4.25 & 0.65 & $87^{\circ}$ & 1.03 & 23 \\
\hline 9304 & L12 & 15.25 & 14.50 & 5.20 & 4.80 & 4.05 & 0.70 & $88^{\circ}$ & 1.05 & 24 \\
\hline 9305 & L2 & 15.40 & 14.70 & 4.70 & 3.10 & 2.65 & 0.70 & $89^{\circ}$ & 1.05 & 24 \\
\hline 9306 & L2 & 15.90 & 17.00 & + & 5.10 & 3.30 & 1.00 & $89^{\circ}$ & 0.94 & 24 \\
\hline 9307 & L2 & 15.90 & 15.40 & + & 5.10 & 2.90 & 0.75 & $88^{\circ}$ & 1.03 & 21 \\
\hline 9308 & L12 & 16.30 & 16.70 & + & 4.80 & 4.45 & 1.00 & $91^{\circ}$ & 0.98 & 24 \\
\hline 9309 & L12 & 16.60 & 17.60 & 5.55 & 4.00 & 4.45 & 0.80 & $90^{\circ}$ & 0.94 & 20 \\
\hline 9310 & L2 & 16.70 & 16.90 & + & 4.05 & 3.05 & 0.80 & $88^{\circ}$ & 0.99 & 23 \\
\hline 9311 & L2 & 16.70 & 17.25 & 5.80 & 4.70 & 2.95 & 0.85 & $94^{\circ}$ & 0.97 & 22 \\
\hline 9312 & L2 & 16.75 & 16.40 & + & 3.65 & 3.60 & 0.85 & $90^{\circ}$ & 1.02 & 23 \\
\hline 9313 & L2 & 17.00 & 17.00 & + & + & 3.60 & + & $90^{\circ}$ & 1.00 & 25 \\
\hline 9314 & L2 & 17.10 & 17.80 & + & + & + & 1.00 & + & 0.96 & + \\
\hline 9315 & L2 & 17.20 & 17.60 & 4.50 & 4.10 & 3.55 & 0.80 & + & 0.98 & 24 \\
\hline 9316 & L2 & 17.20 & 17.65 & + & 4.30 & 3.65 & 0.85 & $90^{\circ}$ & 0.97 & 22 \\
\hline 9317 & $\mathrm{~L} 2$ & 17.25 & 17.30 & 4.40 & 3.50 & 3.70 & 0.80 & $98^{\circ}$ & 1.00 & 24 \\
\hline 9318 & L2 & 17.40 & 17.00 & 5.20 & 4.20 & 4.60 & 1.00 & $87^{\circ}$ & 1.02 & 23 \\
\hline 9319 & L2 & 17.40 & 17.60 & 5.30 & 5.20 & 3.70 & 1.00 & $92^{\circ}$ & 0.99 & 24 \\
\hline 9320 & L2 & 17.65 & 17.40 & 5.60 & 3.60 & 5.60 & 1.00 & $90^{\circ}$ & 1.01 & 24 \\
\hline 9321 & L2 & 17.80 & 18.10 & 5.10 & 3.60 & 4.10 & + & $90^{\circ}$ & 0.98 & 20 \\
\hline 9322 & L12 & 17.80 & 17.60 & 6.00 & 5.60 & 3.20 & 1.05 & $91^{\circ}$ & 1.01 & 23 \\
\hline 9323 & L12 & 18.20 & 20.30 & + & 5.10 & 4.50 & 0.85 & $88^{\circ}$ & 0.90 & 23 \\
\hline 9324 & $\mathrm{~L} 2$ & 18.25 & 19.40 & 7.00 & 4.95 & 3.80 & 0.80 & $93^{\circ}$ & 0.94 & 23 \\
\hline 9325 & L2 & 18.35 & 18.70 & + & 5.10 & 5.20 & 0.98 & $95^{\circ}$ & 0.98 & 23 \\
\hline 9326 & L2 & 18.55 & 19.40 & 5.40 & 4.30 & 4.75 & 0.90 & $89^{\circ}$ & 0.96 & 22 \\
\hline 9327 & L2 & 18.60 & 18.25 & 5.65 & 4.05 & 3.60 & 1.00 & $89^{\circ}$ & 1.02 & 22 \\
\hline 9328 & L2 & 18.85 & 19.80 & 6.00 & 4.30 & 3.95 & 0.85 & $92^{\circ}$ & 0.95 & 21 \\
\hline 9329 & L2 & 18.90 & 18.00 & 5.15 & 4.80 & 4.10 & 0.80 & $91^{\circ}$ & 1.05 & 25 \\
\hline 9330 & L12 & 19.00 & 20.50 & 5.80 & 4.80 & 4.75 & 1.00 & $92^{\circ}$ & 0.93 & 22 \\
\hline 9331 & L12 & 19.00 & 22.60 & + & + & + & 1.00 & + & 0.84 & + \\
\hline 9332 & $\mathrm{~L} 2$ & 19.10 & 20.00 & 4.40 & 3.85 & 4.40 & 0.85 & $95^{\circ}$ & 0.96 & 22 \\
\hline 9333 & L2 & 19.30 & 18.10 & 6.50 & + & 3.85 & 0.80 & $91^{\circ}$ & 1.07 & 21 \\
\hline 9334 & L12 & 19.30 & 19.05 & 7.05 & 5.60 & 4.70 & 1.00 & $91^{\circ}$ & 1.01 & 24 \\
\hline 9335 & $\mathrm{~L} 2$ & 19.50 & 20.80 & + & + & 4.50 & 0.90 & $88^{\circ}$ & 0.92 & 25 \\
\hline 9336 & L2 & 19.55 & 19.85 & 6.05 & 4.60 & 4.20 & 0.90 & $89^{\circ}$ & 0.98 & 21 \\
\hline 9337 & L12 & 19.95 & 21.30 & 7.05 & 5.60 & 4.70 & 1.30 & $91^{\circ}$ & 0.94 & 24 \\
\hline 9338 & L2 & 19.95 & 19.50 & 4.70 & 3.70 & 3.70 & 1.00 & $90^{\circ}$ & 1.02 & 22 \\
\hline 9339 & L2 & 20.00 & 22.00 & 6.70 & 3.60 & 5.40 & 1.10 & $92^{\circ}$ & 0.91 & 26 \\
\hline 9340 & L2 & 20.20 & 21.20 & 5.20 & 4.10 & 5.65 & + & $88^{\circ}$ & 0.95 & 23 \\
\hline 9341 & $\mathrm{~L} 2$ & 20.20 & 18.60 & 5.40 & 3.80 & 5.50 & 0.90 & $83^{\circ}$ & 1.12 & 25 \\
\hline 9342 & L2 & 20.40 & 21.80 & + & 5.20 & 5.10 & 0.90 & $92^{\circ}$ & 0.94 & 25 \\
\hline 9343 & L2 & 20.45 & 21.80 & 7.40 & 5.30 & 5.70 & 1.20 & $91^{\circ}$ & 0.94 & 26 \\
\hline
\end{tabular}


(Table 1 continued)

\begin{tabular}{|c|c|c|c|c|c|c|c|c|c|c|}
\hline Reg. No. & Loc. & $\mathrm{H}$ & $\mathrm{W}$ & $\mathrm{E}_{1}$ & $\mathbf{E}_{2}$ & $\mathrm{D}$ & $\mathrm{C}$ & A.A. & $\mathrm{H} / \mathrm{W}$ & N.R. \\
\hline GK-L9344 & L12 & 20.50 & 22.75 & + & + & 5.45 & 1.05 & $91^{\circ}$ & 0.90 & 24 \\
\hline 9345 & $\mathrm{~L} 2$ & 20.60 & 22.65 & 7.90 & 5.50 & 5.90 & 1.30 & $91^{\circ}$ & 0.91 & 25 \\
\hline 9346 & L2 & 20.65 & 20.20 & 5.70 & 3.60 & 4.10 & 1.00 & $88^{\circ}$ & 1.02 & 23 \\
\hline 9347 & L2 & 20.75 & 22.40 & 7.60 & 5.80 & 4.55 & 0.90 & $89^{\circ}$ & 0.93 & 23 \\
\hline 9348 & L2 & 21.00 & 21.90 & + & 5.70 & 4.90 & 1.00 & $87^{\circ}$ & 0.96 & 23 \\
\hline 9349 & L12 & 21.50 & 23.10 & 9.50 & 6.80 & 5.00 & 1.20 & $90^{\circ}$ & 0.93 & 24 \\
\hline 9350 & $\mathrm{~L} 2$ & 21.60 & 23.10 & 8.55 & 5.50 & 5.80 & 1.00 & $91^{\circ}$ & 0.94 & 24 \\
\hline 9351 & L2 & 21.70 & 22.00 & 7.00 & 5.30 & 5.20 & 1.20 & $89^{\circ}$ & 0.99 & 26 \\
\hline 9352 & L2 & 21.70 & 20.60 & 5.00 & 3.80 & 4.10 & + & $88^{\circ}$ & 1.05 & 23 \\
\hline 9353 & L2 & 21.80 & 24.25 & 7.85 & 6.00 & 6.10 & 1.20 & $88^{\circ}$ & 0.89 & 23 \\
\hline 9354 & L2 & 22.00 & 24.95 & + & + & 5.50 & 1.00 & $92^{\circ}$ & 0.88 & 26 \\
\hline 9355 & L2 & 22.25 & 23.40 & 6.45 & 4.90 & 3.00 & + & $92^{\circ}$ & 0.95 & 25 \\
\hline 9356 & L2 & 22.60 & 25.70 & 8.60 & 5.05 & 6.50 & 1.20 & $90^{\circ}$ & 0.88 & 25 \\
\hline 9357 & L2 & 23.00 & 25.25 & 8.75 & 6.40 & 7.65 & 1.40 & $89^{\circ}$ & 0.91 & 23 \\
\hline 9358 & L2 & 23.20 & 24.15 & + & 6.10 & 4.45 & 1.25 & $91^{\circ}$ & 0.96 & 22 \\
\hline 9359 & L12 & 23.20 & 23.40 & + & 5.40 & 5.20 & + & $86^{\circ}$ & 0.99 & 24 \\
\hline 9360 & L2 & 23.60 & 25.60 & 7.30 & + & 4.60 & 1.30 & + & 0.92 & + \\
\hline 9361 & L2 & 23.80 & 22.30 & 6.70 & 5.70 & 5.70 & 1.30 & $82^{\circ}$ & 1.07 & 22 \\
\hline 9362 & L2 & 24.10 & 27.10 & 6.60 & 5.90 & 5.70 & 1.20 & $92^{\circ}$ & 0.89 & 23 \\
\hline 9363 & L2 & 24.25 & 26.80 & 7.90 & + & 5.50 & 1.00 & $91^{\circ}$ & 0.90 & 22 \\
\hline 9364 & L2 & 24.40 & 27.90 & 9.10 & 5.85 & 9.10 & 1.10 & $92^{\circ}$ & 0.87 & 23 \\
\hline 9365 & L2 & 24.55 & 26.40 & + & 5.80 & 5.80 & 1.20 & $91^{\circ}$ & 0.93 & 23 \\
\hline 9366 & L2 & 24.75 & 26.60 & 7.50 & 4.35 & 6.00 & 1.05 & $88^{\circ}$ & 0.93 & 22 \\
\hline 9367 & L2 & 25.00 & 27.65 & + & 6.60 & 5.60 & 1.25 & $92^{\circ}$ & 0.90 & 23 \\
\hline 9368 & L2 & 25.10 & 26.80 & 9.30 & 6.80 & 7.00 & 1.00 & $91^{\circ}$ & 0.94 & 22 \\
\hline 9369 & L2 & 25.10 & 28.05 & 8.90 & + & 5.75 & + & $90^{\circ}$ & 0.89 & 22 \\
\hline 9370 & L12 & 25.30 & 27.20 & 8.40 & 6.05 & 5.90 & 1.10 & $91^{\circ}$ & 0.93 & 23 \\
\hline 9371 & L2 & 25.65 & 27.30 & + & + & 4.90 & 1.25 & $90^{\circ}$ & 0.94 & 23 \\
\hline 9371 & L2 & 25.65 & 27.30 & + & + & 4.90 & 1.25 & $90^{\circ}$ & 0.94 & 23 \\
\hline 9372 & L2 & 26.40 & 25.65 & + & 6.20 & 5.40 & 1.05 & $78^{\circ}$ & 1.03 & 23 \\
\hline 9373 & L2 & 26.90 & 38.90 & 7.10 & + & 3.95 & 1.25 & $90^{\circ}$ & 0.69 & 22 \\
\hline 9374 & L9 & 27.00 & 29.45 & 8.00 & 5.20 & 6.20 & + & $91^{\circ}$ & 0.92 & 24 \\
\hline 9375 & L2 & 27.05 & 28.75 & + & 5.70 & 6.55 & 1.25 & $86^{\circ}$ & 0.94 & 22 \\
\hline 9376 & L2 & 27.10 & 27.30 & + & 4.90 & 4.85 & 1.10 & $89^{\circ}$ & 0.99 & 24 \\
\hline 9377 & L2 & 28.00 & 28.95 & 6.70 & 5.80 & 6.25 & 1.10 & $90^{\circ}$ & 0.97 & 25 \\
\hline 9378 & L2 & 28.10 & 30.50 & 9.70 & + & 4.55 & 1.30 & + & 0.92 & + \\
\hline 9379 & L18 & 28.15 & 27.40 & + & 6.00 & + & 1.25 & + & 1.03 & 22 \\
\hline 9380 & L2 & 28.20 & 33.65 & 9.30 & + & 9.30 & 1.10 & $88^{\circ}$ & 0.84 & 24 \\
\hline 9381 & L2 & 28.40 & 29.20 & 8.05 & 5.50 & 5.25 & 1.00 & $86^{\circ}$ & 0.97 & 23 \\
\hline 9382 & L2 & 29.30 & 34.60 & 9.50 & + & 7.90 & 1.25 & $91^{\circ}$ & 0.85 & 23 \\
\hline 9383 & L2 & 29.60 & 33.00 & 9.95 & 7.15 & 6.60 & 1.30 & $89^{\circ}$ & 0.90 & 23 \\
\hline 9384 & L2 & 29.80 & 31.60 & 8.00 & 5.40 & 7.75 & 1.40 & $89^{\circ}$ & 0.94 & 23 \\
\hline 9385 & L2 & 30.00 & 30.65 & 8.70 & 6.80 & 7.00 & 1.10 & $90^{\circ}$ & 0.98 & 24 \\
\hline 9386 & L9 & 30.10 & 31.10 & + & 6.80 & 7.90 & + & + & 0.97 & + \\
\hline 9387 & L2 & 31.10 & 32.20 & 9.75 & 4.95 & 6.10 & 1.10 & $94^{\circ}$ & 0.97 & 24 \\
\hline 9388 & L2 & 32.90 & 37.90 & 10.80 & 9.60 & 7.20 & 1.25 & $92^{\circ}$ & 0.87 & 24 \\
\hline 9389 & L2 & 38.10 & 41.20 & + & 6.85 & 7.30 & 1.60 & $90^{\circ}$ & 0.92 & 24 \\
\hline 9526 & $\mathrm{MZ}$ & 11.50 & 11.80 & + & + & + & + & + & 0.94 & 21 \\
\hline 9527 & MZ & 17.60 & 18.80 & 6.20 & 4.80 & + & + & + & 0.94 & 21 \\
\hline 9528 & MZ & 28.00 & 29.00 & + & + & + & + & + & 0.97 & 21 \\
\hline 9529 & BG & 10.15 & 9.40 & 4.20 & 3.10 & 3.20 & + & $96^{\circ}$ & 1.08 & 23 \\
\hline
\end{tabular}


(Table 1 continued)

\begin{tabular}{rcccccccccc}
\hline Reg. No. & Loc. & $\mathrm{H}$ & $\mathrm{W}$ & $\mathrm{E}_{1}$ & $\mathrm{E}_{2}$ & $\mathrm{D}$ & $\mathrm{C}$ & A.A. & $\mathrm{H} / \mathrm{W}$ & N.R. \\
\hline GK-L9530 & BG & 11.90 & 11.85 & 5.00 & 3.85 & + & + & + & 1.00 & 20 \\
9531 & BG & 13.00 & 12.80 & + & 3.85 & + & + & + & 1.02 & + \\
9532 & BG & 13.15 & 12.70 & 4.70 & 2.80 & 2.80 & + & $89^{\circ}$ & 1.04 & 23 \\
9533 & BG & 15.30 & 15.30 & + & 3.60 & + & + & + & 1.00 & + \\
9534 & BG & + & 16.90 & 4.45 & 4.10 & + & + & + & + & 23 \\
9535 & BG & 17.20 & 18.10 & 6.60 & 4.55 & + & + & + & 0.95 & 20 \\
\hline
\end{tabular}

Table 2. Measurements of Cryptopecten yanagawaensis (NomURA and ZiNBo). Left valve.

\begin{tabular}{|c|c|c|c|c|c|c|c|c|c|c|}
\hline Reg. No. & Loc. & $\mathrm{H}$ & W & $E_{1}$ & $\mathrm{E}_{2}$ & D & $\mathrm{C}$ & A.A. & $\mathrm{H} / \mathrm{W}$ & N.R. \\
\hline GK-L9390 & $\mathrm{L} 2$ & 8.80 & 8.10 & 2.90 & 2.40 & 1.40 & 0.40 & $91^{\circ}$ & 1.09 & 23 \\
\hline 9391 & $\mathrm{~L} 2$ & 11.70 & 10.10 & 2.65 & + & 2.15 & 0.60 & $90^{\circ}$ & 1.16 & 23 \\
\hline 9392 & $\mathrm{~L} 2$ & 12.20 & 11.20 & 4.15 & 2.50 & 2.30 & 0.65 & $87^{\circ}$ & 1.09 & 23 \\
\hline 9393 & L12 & 12.40 & 12.70 & 5.15 & 3.80 & 2.30 & 0.55 & $90^{\circ}$ & 0.98 & 22 \\
\hline 9394 & $\mathrm{~L} 2$ & 12.60 & 11.70 & + & + & 2.30 & 0.80 & $89^{\circ}$ & 1.08 & 22 \\
\hline 9395 & L2 & 13.10 & 13.60 & 5.10 & + & 3.00 & 0.50 & $89^{\circ}$ & 0.96 & 24 \\
\hline 9396 & L2 & 13.80 & 13.80 & 4.80 & 3.50 & 3.20 & 0.85 & $95^{\circ}$ & 1.00 & 22 \\
\hline 9397 & L12 & 13.80 & 13.60 & 3.80 & + & 2.90 & 0.75 & $88^{\circ}$ & 1.02 & 22 \\
\hline 9398 & L2 & 14.10 & 14.55 & 5.80 & 3.40 & 3.00 & 0.75 & $92^{\circ}$ & 0.97 & 23 \\
\hline 9399 & $\mathrm{~L} 2$ & 14.10 & 14.90 & 5.00 & + & 2.80 & 1.00 & $93^{\circ}$ & 0.95 & 22 \\
\hline 9400 & L2 & 14.40 & 14.40 & 5.70 & 3.35 & 3.30 & 0.75 & $91^{\circ}$ & 1.00 & 24 \\
\hline 9401 & $\mathrm{~L} 2$ & 14.60 & 14.40 & 4.90 & 3.35 & 2.90 & 0.70 & $93^{\circ}$ & 1.01 & 23 \\
\hline 9402 & L2 & 14.60 & 13.40 & 5.20 & 2.90 & 2.80 & 0.60 & $91^{\circ}$ & 1.09 & 22 \\
\hline 9403 & L2 & 14.70 & 14.20 & 4.60 & 3.70 & 2.50 & 0.80 & $94^{\circ}$ & 1.04 & 22 \\
\hline 9404 & $\mathrm{~L} 2$ & 14.80 & 13.80 & 4.95 & 3.10 & 2.55 & 0.80 & $88^{\circ}$ & 1.07 & 23 \\
\hline 9405 & L12 & 14.80 & 14.70 & 5.30 & 3.90 & 2.10 & 0.70 & $90^{\circ}$ & 1.01 & 21 \\
\hline 9406 & $\mathrm{~L} 2$ & 15.10 & 15.70 & 4.40 & 2.80 & 3.60 & 0.85 & + & 0.96 & 24 \\
\hline 9407 & $\mathrm{~L} 12$ & 15.30 & 9.55 & 4.40 & + & 1.95 & 0.70 & $89^{\circ}$ & 1.60 & 23 \\
\hline 9408 & L12 & 15.60 & 15.80 & 4.55 & + & 3.35 & 0.85 & $90^{\circ}$ & 0.99 & 23 \\
\hline 9409 & $\mathrm{~L} 2$ & 15.70 & 15.90 & + & + & 3.40 & 0.90 & $93^{\circ}$ & 0.99 & 23 \\
\hline 9410 & L18 & 15.80 & 16.30 & 5.65 & 3.65 & 3.40 & + & $91^{\circ}$ & 0.96 & 24 \\
\hline 9411 & L9 & 15.80 & 16.20 & + & 4.70 & 2.90 & 0.75 & + & 0.98 & + \\
\hline 9412 & L18 & 16.00 & 15.00 & 4.60 & 3.80 & 2.60 & + & $88^{\circ}$ & 1.07 & 26 \\
\hline 9413 & L2 & 16.45 & 17.30 & 5.80 & 3.80 & 2.90 & 0.70 & $90^{\circ}$ & 0.95 & 23 \\
\hline 9414 & L2 & 16.85 & 17.05 & 5.15 & 3.70 & 3.40 & 0.85 & $91^{\circ}$ & 0.99 & 22 \\
\hline 9415 & L12 & 17.00 & 17.20 & 4.60 & + & 3.10 & 0.99 & $92^{\circ}$ & 0.99 & 23 \\
\hline 9416 & L12 & 17.30 & 17.45 & 6.00 & 4.55 & 3.15 & 1.00 & $90^{\circ}$ & 0.99 & 22 \\
\hline 9417 & L2 & 17.50 & 19.10 & 6.40 & 4.40 & 3.50 & 1.00 & $95^{\circ}$ & 0.92 & 23 \\
\hline 9418 & L2 & 17.65 & 17.65 & 5.00 & 3.10 & 2.90 & 0.85 & $91^{\circ}$ & 1.00 & 22 \\
\hline 9419 & $\mathrm{~L} 2$ & 17.80 & 18.00 & 5.80 & 2.90 & 3.40 & 0.90 & $91^{\circ}$ & 0.99 & 22 \\
\hline 9420 & L2 & 18.00 & 17.40 & 6.60 & 4.15 & 4.00 & 0.80 & $91^{\circ}$ & 1.03 & 22 \\
\hline 9421 & L2 & 18.00 & 18.35 & 6.00 & + & 4.60 & 1.05 & $92^{\circ}$ & 1.08 & 22 \\
\hline 9422 & L2 & 18.10 & 16.80 & 5.50 & 3.35 & 4.20 & 0.70 & $90^{\circ}$ & 1.08 & 22 \\
\hline 9423 & L12 & 18.10 & 19.80 & + & 5.30 & 3.60 & 1.00 & $92^{\circ}$ & 0.91 & 22 \\
\hline 9424 & L12 & 18.35 & 18.10 & 6.40 & 5.20 & 4.00 & 1.10 & $89^{\circ}$ & 1.00 & 23 \\
\hline 9425 & L2 & 18.40 & 20.40 & + & + & 4.40 & 0.95 & $102^{\circ}$ & 0.90 & 21 \\
\hline 9426 & L2 & 18.40 & 18.35 & 5.30 & 4.20 & 3.70 & 1.00 & $92^{\circ}$ & 1.01 & 22 \\
\hline 9427 & L2 & 18.40 & 20.20 & 6.00 & 5.00 & 4.80 & 0.80 & $92^{\circ}$ & 0.91 & + \\
\hline
\end{tabular}


(Table 2 continued)

\begin{tabular}{|c|c|c|c|c|c|c|c|c|c|c|}
\hline Reg. No. & Loc. & $\mathrm{H}$ & W & $\mathrm{E}_{1}$ & $\mathrm{E}_{2}$ & D & $\mathrm{C}$ & A.A. & $\mathrm{H} / \mathrm{W}$ & N.R. \\
\hline GK-L9428 & $\mathrm{L} 2$ & 18.60 & 18.85 & 6.90 & 5.50 & 4.20 & 1.00 & $95^{\circ}$ & 0.99 & 23 \\
\hline 9429 & L12 & 18.60 & 19.20 & 6.40 & 4.60 & 2.90 & 0.75 & $90^{\circ}$ & 0.97 & 21 \\
\hline 9430 & L2 & 18.80 & 17.80 & 5.20 & 3.80 & 3.80 & 0.85 & $91^{\circ}$ & 1.06 & 22 \\
\hline 9431 & L12 & 18.80 & 18.90 & 6.90 & 4.45 & 3.40 & 1.00 & $91^{\circ}$ & 0.99 & 23 \\
\hline 9432 & L2 & 18.85 & 17.80 & 5.10 & 3.60 & 3.40 & 0.90 & $91^{\circ}$ & 1.06 & 21 \\
\hline 9433 & L12 & 18.95 & 19.30 & 6.30 & 3.80 & 4.10 & 1.20 & $89^{\circ}$ & 0.98 & 22 \\
\hline 9434 & L12 & 19.10 & 20.10 & + & 6.10 & 3.90 & 1.00 & $92^{\circ}$ & 0.95 & 24 \\
\hline 9435 & L12 & 19.10 & 19.20 & + & 3.80 & 3.20 & 0.90 & $88^{\circ}$ & 0.99 & 23 \\
\hline 9436 & $\mathrm{~L} 2$ & 19.10 & 19.80 & 5.80 & 3.40 & 4.40 & 1.00 & $94^{\circ}$ & 0.96 & 22 \\
\hline 9437 & L12 & 19.70 & 21.10 & 7.50 & 5.80 & 4.05 & 1.05 & $91^{\circ}$ & 0.93 & 24 \\
\hline 9438 & L2 & 19.80 & 20.40 & 6.60 & 5.00 & 4.10 & 1.00 & $90^{\circ}$ & 0.97 & 22 \\
\hline 9439 & $\mathrm{~L} 12$ & 20.00 & 20.80 & 5.50 & 4.00 & 4.80 & 1.25 & $88^{\circ}$ & 0.96 & + \\
\hline 9440 & L18 & 20.00 & 20.50 & 7.15 & 4.60 & 4.20 & 1.10 & $88^{\circ}$ & 0.98 & 21 \\
\hline 9441 & L2 & 20.00 & 19.50 & 6.00 & 4.40 & 3.70 & 1.05 & $89^{\circ}$ & 1.03 & 22 \\
\hline 9442 & L2 & 20.10 & 20.50 & 5.25 & 4.10 & 4.70 & 1.00 & $92^{\circ}$ & 0.98 & 22 \\
\hline 9443 & L2 & 20.10 & 20.15 & 6.35 & + & 4.30 & + & $86^{\circ}$ & 1.00 & 19 \\
\hline 9444 & L12 & 20.30 & 20.20 & 5.70 & 4.00 & 4.90 & 1.20 & $91^{\circ}$ & 1.00 & 25 \\
\hline 9445 & L12 & 20.40 & 20.50 & + & 4.60 & 5.00 & 1.00 & $90^{\circ}$ & 1.00 & 23 \\
\hline 9446 & L12 & 20.45 & 20.40 & 6.10 & 4.10 & 3.40 & 1.10 & $91^{\circ}$ & 1.00 & 23 \\
\hline 9447 & L2 & 20.50 & 20.20 & 5.20 & 4.20 & 4.50 & 1.00 & $99^{\circ}$ & 1.01 & 23 \\
\hline 9448 & L12 & 20.50 & 20.60 & 6.70 & 4.70 & 4.00 & 1.00 & $88^{\circ}$ & 1.00 & 23 \\
\hline 9449 & L2 & 20.60 & 20.30 & 5.80 & 4.40 & 4.50 & 1.20 & $91^{\circ}$ & 1.01 & 23 \\
\hline 9450 & $\mathrm{~L} 2$ & 20.65 & 22.05 & 7.00 & 6.20 & 4.70 & 1.05 & $96^{\circ}$ & 0.94 & 23 \\
\hline 9451 & $\mathrm{~L} 2$ & 20.75 & 20.80 & 5.10 & 3.45 & 4.15 & 1.00 & $94^{\circ}$ & 1.00 & 23 \\
\hline 9452 & L2 & 20.80 & 22.00 & 6.70 & 5.40 & 4.20 & 1.30 & $93^{\circ}$ & 0.95 & 25 \\
\hline 9453 & L12 & 20.85 & 21.40 & 7.60 & 4.10 & 4.50 & 1.10 & $96^{\circ}$ & 0.97 & 22 \\
\hline 9454 & L2 & 20.90 & 21.00 & 6.45 & 3.75 & 4.00 & 1.20 & $93^{\circ}$ & 1.00 & 22 \\
\hline 9455 & L12 & 21.10 & 20.80 & 7.30 & 4.35 & 4.05 & 1.00 & $88^{\circ}$ & 1.01 & 23 \\
\hline 9456 & L2 & 21.40 & 22.20 & 7.55 & 4.55 & + & 1.30 & $96^{\circ}$ & 0.96 & 23 \\
\hline 9457 & L2 & 21.40 & 21.80 & 6.80 & 5.25 & 4.20 & 1.00 & $93^{\circ}$ & 0.98 & 26 \\
\hline 9458 & L2 & 21.40 & 22.20 & 6.80 & 4.50 & 4.00 & 1.00 & $92^{\circ}$ & 0.96 & 23 \\
\hline 9459 & $\mathrm{~L} 2$ & 21.60 & 24.55 & 8.10 & 5.85 & 3.80 & 1.25 & $91^{\circ}$ & 0.88 & 21 \\
\hline 9460 & L12 & 21.60 & 23.10 & 7.30 & 4.70 & 3.50 & 1.00 & $95^{\circ}$ & 0.94 & 22 \\
\hline 9461 & L12 & 22.20 & 24.80 & + & 5.30 & 3.40 & 1.00 & $92^{\circ}$ & 0.90 & 23 \\
\hline 9462 & L12 & 22.30 & 23.40 & 7.60 & 5.50 & 5.25 & 1.20 & $91^{\circ}$ & 0.95 & 21 \\
\hline 9463 & L2 & 22.40 & 22.40 & 6.85 & 5.60 & 4.80 & 1.10 & $92^{\circ}$ & 1.00 & 23 \\
\hline 9464 & $\mathrm{~L} 2$ & 22.40 & 22.40 & 6.20 & 4.70 & 4.60 & 0.80 & $92^{\circ}$ & 1.00 & 21 \\
\hline 9465 & L12 & 22.50 & 25.05 & 7.90 & 5.85 & 4.95 & 1.00 & $94^{\circ}$ & 0.90 & 22 \\
\hline 9466 & L2 & 22.60 & 24.00 & 7.60 & 5.50 & 4.20 & 0.85 & $93^{\circ}$ & 0.94 & 23 \\
\hline 9467 & L12 & 22.70 & 25.00 & 6.90 & 6.20 & 5.25 & 1.00 & $97^{\circ}$ & 0.91 & 23 \\
\hline 9468 & L2 & 22.90 & 24.40 & 5.80 & + & 6.30 & 1.05 & $93^{\circ}$ & 0.94 & 24 \\
\hline 9469 & L2 & 23.00 & 24.20 & 7.00 & 5.00 & 5.50 & 1.20 & $86^{\circ}$ & 0.95 & 22 \\
\hline 9470 & $\mathrm{~L} 2$ & 23.20 & 24.60 & 7.90 & 6.00 & 5.60 & 1.00 & $93^{\circ}$ & 0.94 & 25 \\
\hline 9471 & L2 & 23.75 & 21.40 & 6.60 & 4.30 & 5.70 & 1.00 & $89^{\circ}$ & 1.11 & 21 \\
\hline 9472 & $\mathrm{~L} 2$ & 24.60 & 24.80 & 7.00 & + & + & 1.25 & + & 0.99 & 23 \\
\hline 9473 & L2 & 24.80 & 25.80 & 9.00 & 6.00 & 4.70 & 1.15 & $100^{\circ}$ & 0.96 & 22 \\
\hline 9474 & L2 & 24.90 & 26.40 & 8.20 & 5.80 & 5.50 & 1.05 & $93^{\circ}$ & 0.94 & 22 \\
\hline 9475 & $\mathrm{~L} 2$ & 25.00 & 26.00 & 6.60 & 5.60 & 4.55 & 1.20 & $95^{\circ}$ & 0.96 & 22 \\
\hline 9476 & L9 & 25.50 & 26.60 & 8.60 & 6.80 & 5.20 & 1.45 & + & 0.96 & 22 \\
\hline 9477 & $\mathrm{~L} 2$ & 25.90 & 27.10 & 9.30 & 6.70 & 5.70 & 1.20 & $96^{\circ}$ & 0.96 & 23 \\
\hline 9478 & L2 & 26.10 & 23.35 & 6.90 & 3.90 & 6.45 & 1.40 & $91^{\circ}$ & 1.12 & 23 \\
\hline
\end{tabular}


(Table 2 continued)

\begin{tabular}{|c|c|c|c|c|c|c|c|c|c|c|}
\hline Reg. No. & Loc. & $\mathrm{H}$ & W & $\mathrm{E}_{1}$ & $\mathrm{E}_{2}$ & $\mathrm{D}$ & $\mathrm{C}$ & A.A. & $\mathrm{H} / \mathrm{W}$ & N.R. \\
\hline GK-L9479 & $\mathrm{L} 2$ & 26.20 & 29.20 & 7.00 & 5.80 & 6.00 & + & $97^{\circ}$ & 0.90 & 24 \\
\hline 9480 & L2 & 26.40 & 27.50 & 8.30 & 5.60 & 6.40 & 1.40 & $100^{\circ}$ & 0.96 & 22 \\
\hline 9481 & L12 & 26.50 & 28.70 & 10.90 & 7.30 & + & 1.40 & + & 0.92 & + \\
\hline 9482 & L2 & 26.70 & 29.00 & 8.35 & 6.20 & 6.30 & 1.45 & $93^{\circ}$ & 0.92 & 23 \\
\hline 9483 & $\mathrm{~L} 2$ & 27.40 & 27.00 & 7.60 & + & 7.20 & 1.35 & + & 1.01 & 23 \\
\hline 9484 & L2 & 27.60 & 30.50 & 8.85 & 5.90 & 5.60 & 1.20 & $100^{\circ}$ & 0.90 & 23 \\
\hline 9485 & L2 & 28.00 & 27.30 & 9.30 & 6.60 & 5.95 & 1.00 & $90^{\circ}$ & 1.03 & 24 \\
\hline 9486 & L2 & 28.10 & 27.60 & 7.60 & 4.40 & 6.60 & 1.25 & $88^{\circ}$ & 1.02 & 22 \\
\hline 9487 & $\mathrm{~L} 2$ & 28.20 & 29.55 & 9.70 & 7.15 & 4.95 & 0.95 & $93^{\circ}$ & 0.95 & 23 \\
\hline 9488 & L2 & 28.20 & 30.40 & 9.00 & 6.50 & 5.10 & 1.10 & $93^{\circ}$ & 0.93 & 23 \\
\hline 9489 & L2 & 28.70 & 30.60 & 9.90 & 7.00 & 6.70 & 1.35 & $100^{\circ}$ & 0.94 & 24 \\
\hline 9490 & L12 & 29.10 & 31.60 & 10.00 & 6.60 & 7.15 & 1.20 & $88^{\circ}$ & 0.94 & 23 \\
\hline 9491 & L2 & 30.00 & 38.50 & 8.90 & 7.20 & 7.45 & 1.40 & $91^{\circ}$ & 0.80 & 22 \\
\hline 9492 & L2 & 30.00 & 33.00 & 9.60 & 6.40 & 6.20 & 1.30 & $87^{\circ}$ & 0.90 & 22 \\
\hline 9493 & $\mathrm{~L} 2$ & 30.20 & 31.80 & 8.90 & 6.10 & 6.80 & 0.95 & $90^{\circ}$ & 0.95 & 24 \\
\hline 9494 & L2 & 30.20 & 34.10 & 10.90 & 6.80 & 8.00 & 1.60 & $89^{\circ}$ & 0.89 & 23 \\
\hline 9495 & L2 & 30.70 & 33.15 & 9.90 & 6.30 & 6.40 & 1.55 & $94^{\circ}$ & 0.93 & 22 \\
\hline 9496 & L2 & 30.70 & 33.30 & 8.20 & 6.20 & 6.80 & 1.40 & $93^{\circ}$ & 0.92 & 23 \\
\hline 9497 & L2 & 31.30 & 32.40 & 7.70 & 5.50 & 6.40 & 1.30 & $92^{\circ}$ & 0.91 & 21 \\
\hline 9498 & L2 & 31.30 & 34.30 & 9.90 & 6.50 & 7.80 & 1.40 & $93^{\circ}$ & 0.91 & 21 \\
\hline 9499 & L2 & 32.40 & 32.00 & 7.20 & 5.80 & 7.40 & 1.45 & $95^{\circ}$ & 0.95 & 22 \\
\hline 9500 & L2 & 32.60 & 34.40 & 7.60 & 5.80 & 5.50 & 1.20 & $93^{\circ}$ & 0.95 & 22 \\
\hline 9501 & L2 & 33.80 & 34.60 & 8.80 & 6.80 & 8.10 & 1.35 & $88^{\circ}$ & 0.98 & 24 \\
\hline 9502 & L2 & 34.40 & 37.00 & 10.15 & 6.30 & 8.60 & 1.30 & $92^{\circ}$ & 0.93 & 24 \\
\hline 9503 & L2 & 34.40 & 39.10 & 9.80 & 7.60 & 8.60 & 1.65 & $96^{\circ}$ & 0.88 & 22 \\
\hline 9504 & L2 & 35.20 & 39.30 & + & + & 7.70 & 1.55 & + & 0.90 & 24 \\
\hline 9505 & L2 & 35.20 & 38.10 & 11.80 & 7.00 & 9.80 & 1.60 & $92^{\circ}$ & 0.93 & 24 \\
\hline 9506 & L2 & 35.35 & 34.80 & 10.10 & 6.30 & 7.00 & 1.25 & + & 1.02 & 25 \\
\hline 9507 & L2 & 35.50 & 35.05 & 9.00 & 6.05 & 8.50 & 1.05 & $96^{\circ}$ & 1.01 & 24 \\
\hline 9508 & L2 & 35.60 & 41.30 & 9.60 & 7.50 & 11.30 & 1.70 & $92^{\circ}$ & 0.86 & 22 \\
\hline 9509 & L2 & 36.60 & + & 9.80 & 7.70 & 8.50 & 1.40 & $94^{\circ}$ & + & 23 \\
\hline 9510 & L2 & 39.40 & 36.60 & 11.20 & 7.15 & 9.00 & 1.50 & $89^{\circ}$ & 1.08 & 24 \\
\hline 9511 & L2 & 40.00 & + & 9.55 & 6.75 & 8.60 & 1.45 & $94^{\circ}$ & + & 23 \\
\hline 9512 & MZ & 16.00 & 16.50 & + & + & + & + & + & 0.97 & 22 \\
\hline 9513 & $\mathrm{MZ}$ & 19.30 & 20.80 & 4.80 & + & + & + & + & 0.93 & 21 \\
\hline 9514 & MZ & 19.30 & 20.30 & 8.20 & 5.50 & + & + & + & 0.95 & 22 \\
\hline 9515 & $\mathrm{MZ}$ & 26.20 & 29.00 & 8.60 & + & + & + & + & 0.90 & 23 \\
\hline 9516 & $\mathrm{MZ}$ & 31.00 & 33.50 & + & + & + & + & + & 0.93 & 24 \\
\hline 9517 & BG & 8.60 & 7.70 & 3.50 & 2.20 & + & + & $91^{\circ}$ & 1.12 & 24 \\
\hline 9518 & BG & 9.40 & 8.80 & + & + & + & + & + & 1.07 & 24 \\
\hline 9519 & BG & 10.70 & 9.60 & 4.45 & + & + & + & + & 1.11 & 23 \\
\hline 9520 & BG & 10.90 & 9.80 & 4.40 & 2.90 & + & + & + & 1.11 & 22 \\
\hline 9521 & $\mathrm{BG}$ & + & 11.80 & + & + & + & + & + & + & + \\
\hline 9522 & BG & 13.40 & 12.50 & 4.35 & + & + & + & + & 1.07 & 23 \\
\hline 9523 & BG & 14.20 & 14.40 & + & 3.90 & + & + & + & 0.86 & + \\
\hline 9524 & BG & 14.55 & 15.30 & 4.50 & 4.00 & + & + & $93^{\circ}$ & 0.95 & 23 \\
\hline 9525 & BG & 16.40 & 18.20 & 6.60 & + & 3.20 & + & $89^{\circ}$ & 0.90 & 23 \\
\hline
\end{tabular}




\section{Cryptopecten yanagawaensis (NomURA and ZINBO)}

(right; pl. 36. figs. 1-17b and left; pl. 37. figs. 1-20)

1936. Pecten (Aequipecten ?) yanagawaensis NomURA and Zinbo, Saito Ho-On Kai Mus. Res. Bull., (10), p. 337, pl. 20, figs. 2a-b.

1940. Pecten (Aequipecten) yanagawaensis, NomUrA, Sci. Rep. Tohoku Imp. Univ., [2], 21, (1), p. 19, pl. 1, figs. 10-13.

1958. Cryptopecten yanagawaensis, MASUdA, Trans. Proc. Paleont. Soc. Japan, N.S., (30), p. 189, pl. 27b, figs. 1-8.

1962. Aequipecten yanagawaensis, MASUDA, Sci. Rep. Tohoku Univ., [2], 33, (2), p. 192, pl. 26, fig. 8 .

1965. Aequipecten yanagawaensis, MASUdA and TAkegaWA, Saito Ho-On Kai Mus. Res. Bull., (40), pl. 1, figs. 12-13.

?1973. Aequipecten (Cryptopecten) yanagawaensis, ShIKAMA, Sci. Rep. Tohoku Univ., [2], Spec. Vol., (6), p. 190, 194. (P. 190, in the Table 2).

1973b. Aequipecten yanagawaensis, MASUdA, Atlas of Japanese Fossils, (33), pl. N-54, figs. 14, 16.

1974. Cryptopecten yanagawaensis, Itorgawa in Itorgawa, Shibata and Nishiмото, Bull. Mizunami Fossil Mus. (1), p. 67, pl. 11, figs. 6-9b.

1976. Aequipecten yanagawaensis, OGASAWARA, Sci. Rep. Tohoku Univ. [2], 46, (2), p. 44, pl. 3, fig. 3,6 .

1979. Cryptopecten yanagawaensis, TAGUChI, ONo and Окамото, Bull. Mizunami Fossil Mus., (6), pl. 4, figs. 1, 2.

1981. Aequipecten yanagawaensis, Itorgawa, Shibata, Nishimoto and OkUmURA, Monogr. Mizunami Fossil Mus., (3A), pl. 7, figs. 2, 3.

1982. Aequipecten yanagawaensis, ITOIGAWA, SHIBATA, Nishimoto and OKUMURA, Monogr. Mizunami Fossil Mus. (3B), p. 46.

1982. Cryptopecten yanagawaensis, HAYAMI, Venus, 41, (3), p. 235.

1984. Cryptopecten yanagawaensis, HaYAMI, Bull. Univ. Mus. Univ. Tokyo, (24), p. 113, pl. 8, figs. 6-9.

Measurements:-Measured parts are shell height $(\mathrm{H})$, shell width (W), apical angle (A.A.). length of anterior $\left(E_{1}\right)$ and posterior ear $\left(E_{2}\right)$ and prominence of radial ribs (C) on both valves. Maximum number of primary radial ribs are also counted (N.R.).

Typology:-Holotype (Left valve) SM, Reg. No. 8353 (Saito Ho-On Kai Museum).

$\begin{array}{cccccccr}\mathrm{H} & \mathrm{W} & \mathrm{E}_{1} & \mathrm{E}_{2} & \mathrm{D} & \text { A.A. } & \mathrm{H} / \mathrm{W} & \text { N.R. } \\ (\mathrm{mm}) & (\mathrm{mm}) & (\mathrm{mm}) & (\mathrm{mm}) & (\mathrm{mm}) & (\text { degree }) & & \\ 10.00 & 21.50 & + & + & 5.00 & 110^{\circ} & 0.93 & +\end{array}$

Type locality and Geological formation:-Cliff of the Hirose River at the southeast end of the Yanagawa Park, Yanagawa-machi Date-gun, Fukushima Prefecture (Lat. $37^{\circ} 51^{\prime} 05^{\prime \prime} \mathrm{N}$, Long. $140^{\circ} 36^{\prime} 05^{\prime \prime} \mathrm{E}$ ).

Material:-See the Table 1 and 2. The number of total individuals is 243 (106 right valves and 137 left ones). Material almost consist of samples from the fossil locality $\mathrm{L} 2$.

Diagnosis:-Shell orbicular, moderate in size and thickness, valves less convexed than those of other species of Cryptopecten, laterally much convexed, with concentric lamellae on both valves and apical angle of about $90^{\circ}$. Byssal notch 
and ctenolium conspicuous. Stout, broad and flat-topped radial ribs with oppositely disposed imbricated scales of about twenty three.

Description:-Shell is moderate in size. While maximum, minimum and mean shell height $(\mathrm{H})$ are $38.10 \mathrm{~mm}, 10.35 \mathrm{~mm}$ and $20.98 \mathrm{~mm}$ respectively, on right valve, they are $40.00 \mathrm{~mm}, 8.80 \mathrm{~mm}$ and $22.29 \mathrm{~mm}$ on left valve. It is moderately thick, suborbicular in outline and nearly equivalve. Right is a little more convex than the left valve in the younger stage. Length of the posterior side is longer than that of the anterior one and inequilateral on both valves in

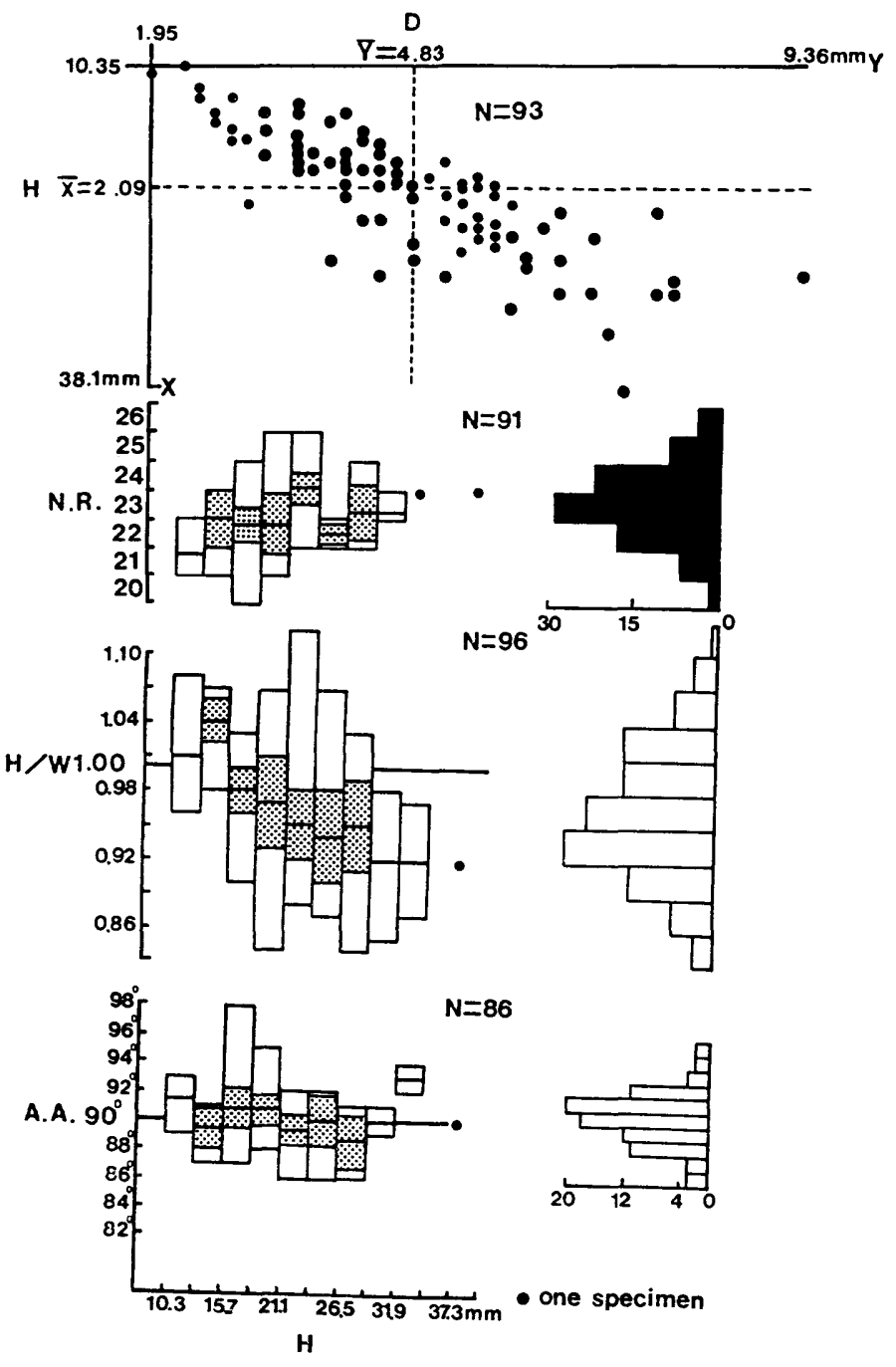

Fig. 3. Variation of apical angle (A.A.), proportion of shell height to width $(\mathrm{H} / \mathrm{W})$ and number of radial ribs (N.R.) and scatter diagram showing relationshop between shell height $(\mathrm{H})$ and shell depth (D) on right valve of Cryptopecten yanagawaensis (NomURA and ZINBO) from the Moniwa Formation. Each bar shows mean value. The dotted rectangles represent 90 percent confidence limits for the mean. 
the adult stage. Ratio of shell height $(\mathrm{H})$ to width (W) clearly changes within growth series (Fig. 3 and 4). Shell-width increases rapidly after shell-height attains $20 \mathrm{~mm}$ on both valves. Posterior submargins are longer than anterior and regularly concave. Apical angle is about $90^{\circ}$, averaging $92^{\circ}$, on left valve and $90^{\circ}$ on right valve. Valves are radiately ribbed. Ribs on right valve are countable about 23 , elevated, more or less squarish in the younger stage and rounded in the adult stage. Ribs are accompanied by a fine imbricated thread each on both sides. On many specimens top of radial ribs is worn and smooth,

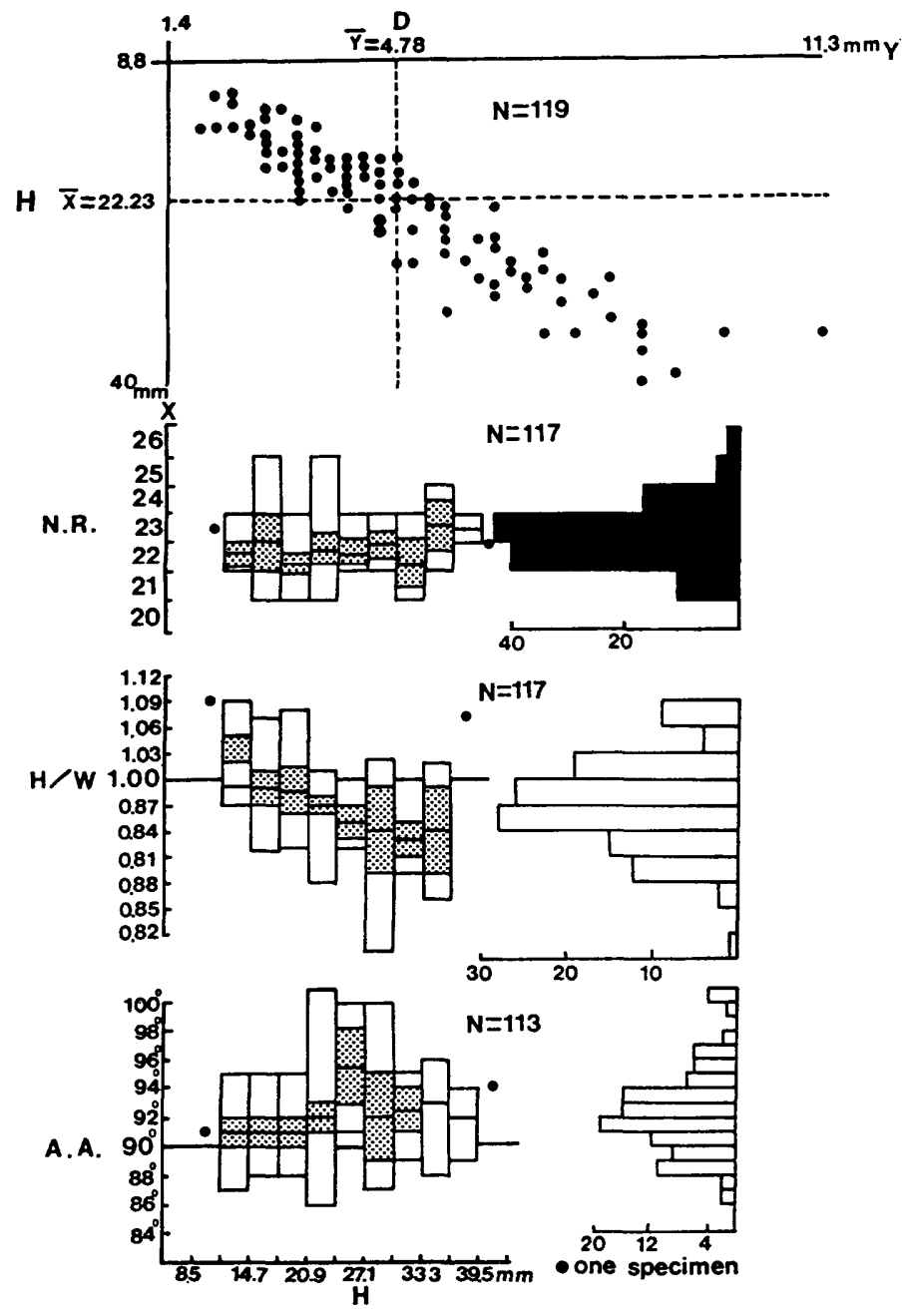

Fig. 4. Variation of apical angle (A.A.), proportion of shell height to width (H/W) and number of radial ribs (N.R.) and scatter diagram showing relationship between shell height $(\mathrm{H})$ and shell depth (D) on left valve of Cryptopecten yanagawaensis (NOMURA and ZINBO) from the Moniwa Formation. Each bar shows mean value. The dotted rectangles represent 90 percent confidence limits for the mean. 
although numerous concentric lamellae are observed on well preserved specimens collected from L 12 and L 18. Interspaces are nearly equal to or a little broader than ribs themselves and sculptured with numerous transverse fine lamellae. Anterior auricle is large than posterior one, sculptured with fine, distinct and imbricated radial ribs and concentric lines, and furnished with deep byssal area. Posterior auricle is triangular and truncated behind at right angle and similar to the anterior one in its sculpture, though radial threads are less distinct than the anterior. Radial ribs are oppositely scaled on both valves. The manner of disposition of scale is the same as that of $C$. vesiculosus. Hinge line is straight. Hinge has narrow but deep resilial pit. Triangular plane and teeth on both sides of resilial pit are distinct. Ctenolium and cardinal crura are conspicuous and ornamented by fine transverse incision. Radial ribs on the left valve have similar sculpture to the right one. Internal surface of both valves is rather smooth except for the characteristic ventral serration (pl. 37. fig. 19.).

Ontogeny:-Though growth rings are rather obscure, three inferred annual rings are discernible, at growth stages with the shell-height of about $20 \mathrm{~mm}$, $25 \mathrm{~mm}$ and $35 \mathrm{~mm}$. Radial ribs are distinct even on the smallest specimens of less than $10 \mathrm{~mm}$ high. Depth of shell (D) increases in proportion to shell height. There is no statistical difference of shell depth between the right and left valves among the specimens larger than $13 \mathrm{~mm}$ high (fig. 20 in pl. 37. and $15 \mathrm{~b}$ in pl. 36.). Byssal notch and ctenolium are distinct in the younger stage but becomes obscure in the adult stage. Proportion of shell height to width changes with growth. It is larger than 1.0 until shell attains to $20 \mathrm{~mm}$ high, then it decreases but becomes stable after attaining $30 \mathrm{~mm}$ in height. Radial rib is square in profile of cross-section in the younger stage and nearly triangular as shell attains above $30 \mathrm{~mm}$ in height. Radial ribs are bipartited respectively by intercalation of a shallow longitudinal furrow each on both valves in the adult stage. Posterior ends of both valves are considerably elongated in the adult stage. Maximum height of commissure-waves in ventral view (C) increases in proportion to shell height (Fig. 7). Fine lateral threads appear on both sides of radial ribs when shell attain $10 \mathrm{~mm}$ high. Interspace between the fine thread and radial rib is decked with slender concentric lamellae to form a series of chambers, although these chambers are usually broken by abrasion except for excellently preserved specimens.

Variation:-MASUDA (1958) reported that the number of radial ribs varies

\section{Explanation of Plate 36}

(All figures are in natural size and all specimens are collected from $L 2$ unless otherwise stated.)

Cryptopecten yanagawaensis (NomURA and ZINBO)

Right valve.
1. GK-L9385.
2. GK-L9394.
3. GK-L9302.
4. GK-L9304 (L 12).
5. GK-L9311.
6. GK-L9319 (L 12). 7. GK-L9326. 8. GK-L9333.
9. GK-L9328.
10. GK-L9352. 11. GK-L9345. 12. GK-L9361.
13. GK-L9366.
14. K-L9381. 15 and b, GK-L9383 $(b, \times 1.2)$.
16. GK-L9385. 17a and b, GK-L9389. 
Mem. Fac. Sci., Kyushu Univ., Ser. D, Vol. XXV

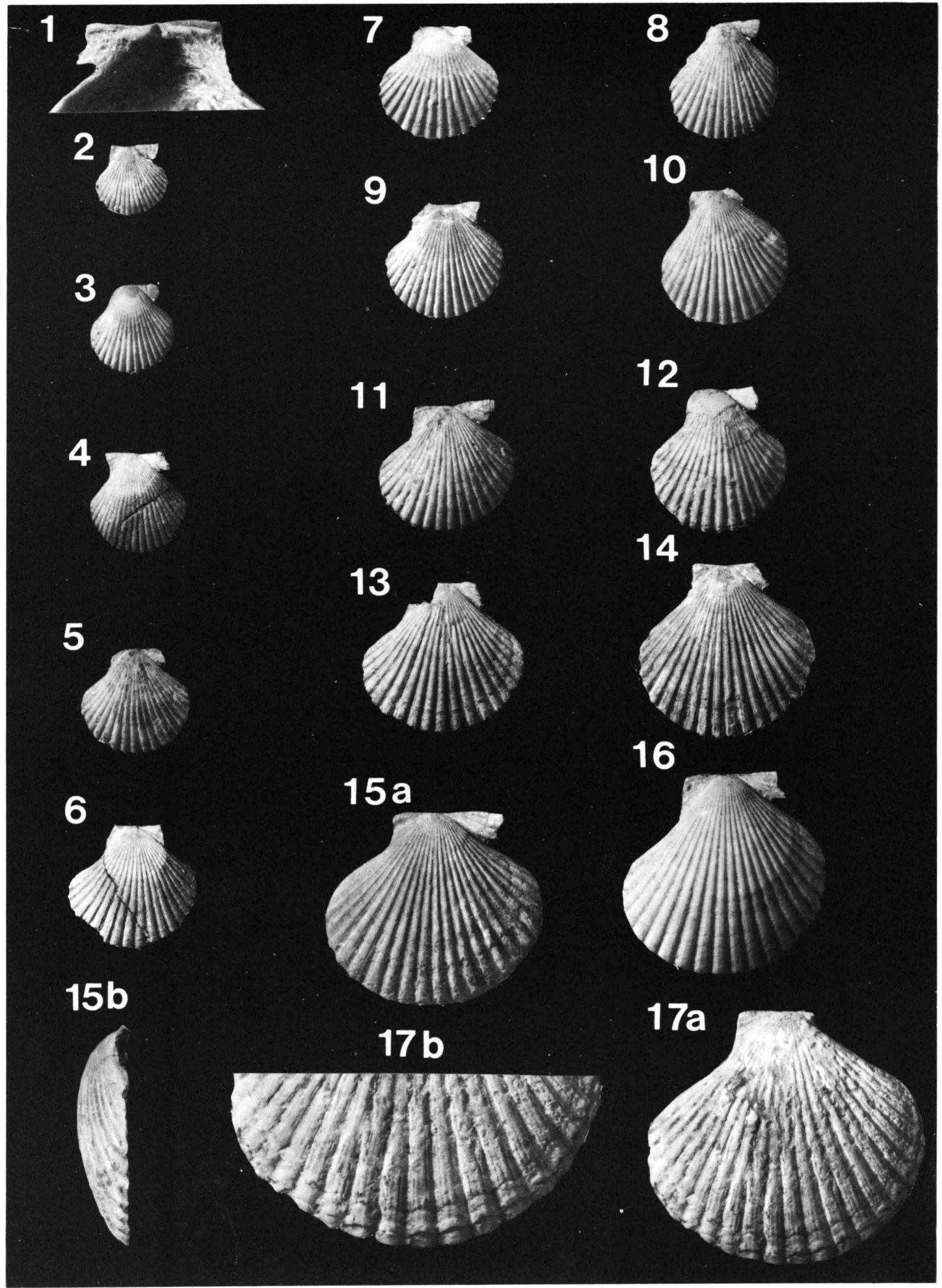

Y. SATo: Redescription of Cryptopecten yanagawaensis 


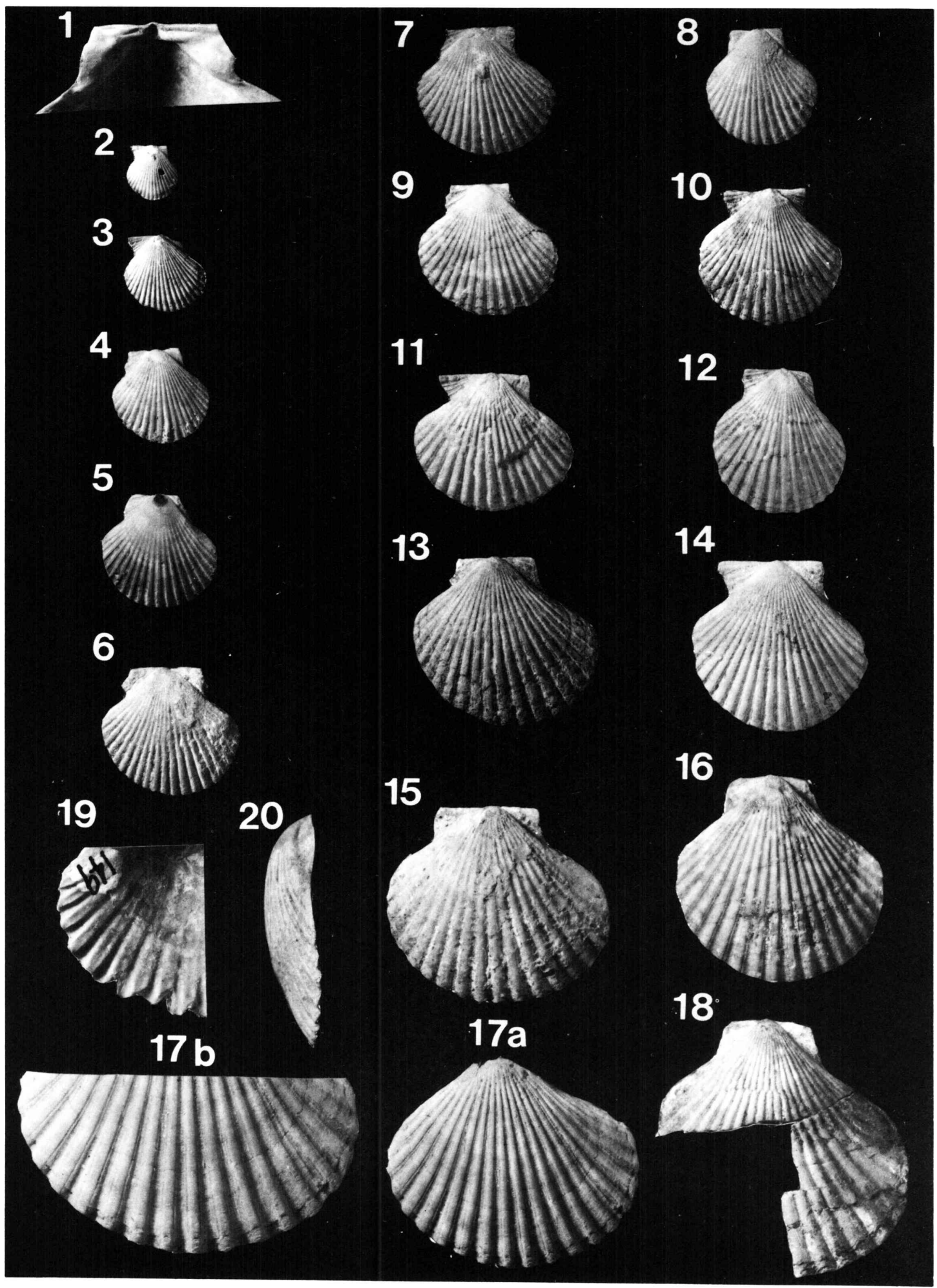

Y. SATo: Redescription of Cryptopecten yanagawaensis 
between 16 and 26 (averaging 21) on both valves, but it ranges from 21 to 26 (averaging 23) on both valves in the present material (Fig. 3 and 4). Its range is nearly equal to that of Recent Cryptopecten vesiculosus. Apical angle (A.A.) is independent of shell height and ranges from $86^{\circ}$ to $101^{\circ}$ (averaging $92^{\circ}$ ) on the right valve and from $86^{\circ}$ to $98^{\circ}$ (averaging $90^{\circ}$ ) on the left valve. As a rule, there is no intercalary rib or thread on the interspaces between radial ribs, but an intercalary thread is rarely found at posterior and anterior ends. The last mentioned type is also found in the specimens collected from the Bihoku Group, Okayama Prefecture. Two phenotypes recognized in the Pliocene and Recent $C$. vesiculosus regarding the relative elevation of radial ribs (HABE and KosUge, 1967 and HAYAMI, 1973 and 1984) can not be observed in the present material of C. yanagawaensis. Shell-width increases more rapidly than shellheight after certain stage of growth as mentioned before, but there is a type with shell height still larger than shell-width even in the adult stage (fig. 15 and 16 in pl. 36 . and fig. $17 \mathrm{a}$ and $18 \mathrm{in} \mathrm{pl.} \mathrm{37.).} \mathrm{Specimens} \mathrm{of} \mathrm{this} \mathrm{type} \mathrm{are} \mathrm{usually}$ observed in those specimens collected from L 2. In this type, however, the other characteristics of morphology are the same as those of the normal type.

Comparison with fossil species:-As pointed out by VoKes (1967 and 1980), Aequipecten FISHER, 1886 was proposed earlier than Crytopecten DALL, BARTSCH and REHDER, 1938. On the basis of fossil material the following species have been reported under the genus Aequipecten in Japan (MASUdA and NoDA, 1976); vesiculosus (YoKоYамA, 1911 and 1922), kyushuensis (NAGAO, 1928), kikaiensis (NomURA and ZINBo, 1934), yanagawaensis (NoMURA and ZINBo, 1936), sematensis (TAKI and OYAMA, 1954), hataii (KANNO, 1958) and matsunagiensis (MASUda, 1966). While HiRAyama (1954) described oyamaensis under the genus Cryptopecten. Among these species, kyushuensis, kikaiensis, sematensis and vesiculosus are considered to belong to Cryptopecten because they posses the characteristic hollow chambers on both sides of radial ribs. C. kyushuensis is distinguishable from yanagawaensis by smaller and more convex shell, and distinctly elevated, round topped radial ribs of smaller number. C. vesiculosus from the Pliocene and Pleistocene formations differs from the present species in having fewer radial ribs accompanied with a imbricated thread on each lateral sides of them and a few number of imbricated intercalary threads on the interspaces of ribs on lower part of disc, and also having flatter left valve. $C$. kikaiensis was included into $n u x$ by Hayami (1984) as subspecies. New species of Cryptopecten, C. spinosus, and two new subspecies of C. vesiculosus, vesiculosus makiyamai and $v$. sematensis, were proposed by HAYAMI (1984). Present

\section{Explanation of Plate 37}

Cryptopecten yanagawaensis (NOMURA and ZINBO)

Left valve.

1. GK-L9479. 2. GK-L9390. 3. GK-L9393. 4. GK-L9400.

5. GK-L9429. 6. GK-L9437 (L 12). 7. GK-L9450. 8. GK-L9432.

9. GK-L9459. 10. K-L9471. 11. GK-L9466. 12. GK-L9457.

13. GK-L9390. 14. GK-L9477. 15 and 20, GK-L9491 $(20, \times 1.2)$.

16. GK-L9499. 17a, b. GK-L9604. 18. GK-L9611, 19. GK-L9490. 
species is distinguishable from makiyamai and spinosus by their larger number of radial ribs than the latter and from sematensis by difference of imbrication which appears in alternative disposition in sematensis. Fossil species of Cryptopecten has not been reported from the the West Coast of North America.

Comparison with recent species:- $\mathrm{HABE}$ (1977) distinguished five living species of Cryptopecten in the Japanese waters. They are vesiculosus, tissotii, nux, owenii and inaequivalvis. Subsequently, HAYAMI (1984) regarded tissoti and alli as synonyms of bullatus and he recognized four species of Cryptopecten, bullatus, nux, vesiculosus and phrygium. C. nux (REEVE) known from the Early Miocene to Recent in the Indo-Pacific is distinguishable from the present species by its smaller and more convex shell. C. bullatus (DATZENBERG and BAVAY) from the Late Pliocene to Recent in the Southeast Asia is also different from the present species by the smaller number of radial ribs and flatter left valve. Fossil and Recent vesiculosus is distinguishable from the present species by its smaller number of radial ribs, intercalary imbricated thread between radial ribs and flatter left valve. C. phrygium (DALL) from the western Atlantic Ocean differs from the present species by its smaller number of radial ribs and illdeveloped byssal notch in the right valve.

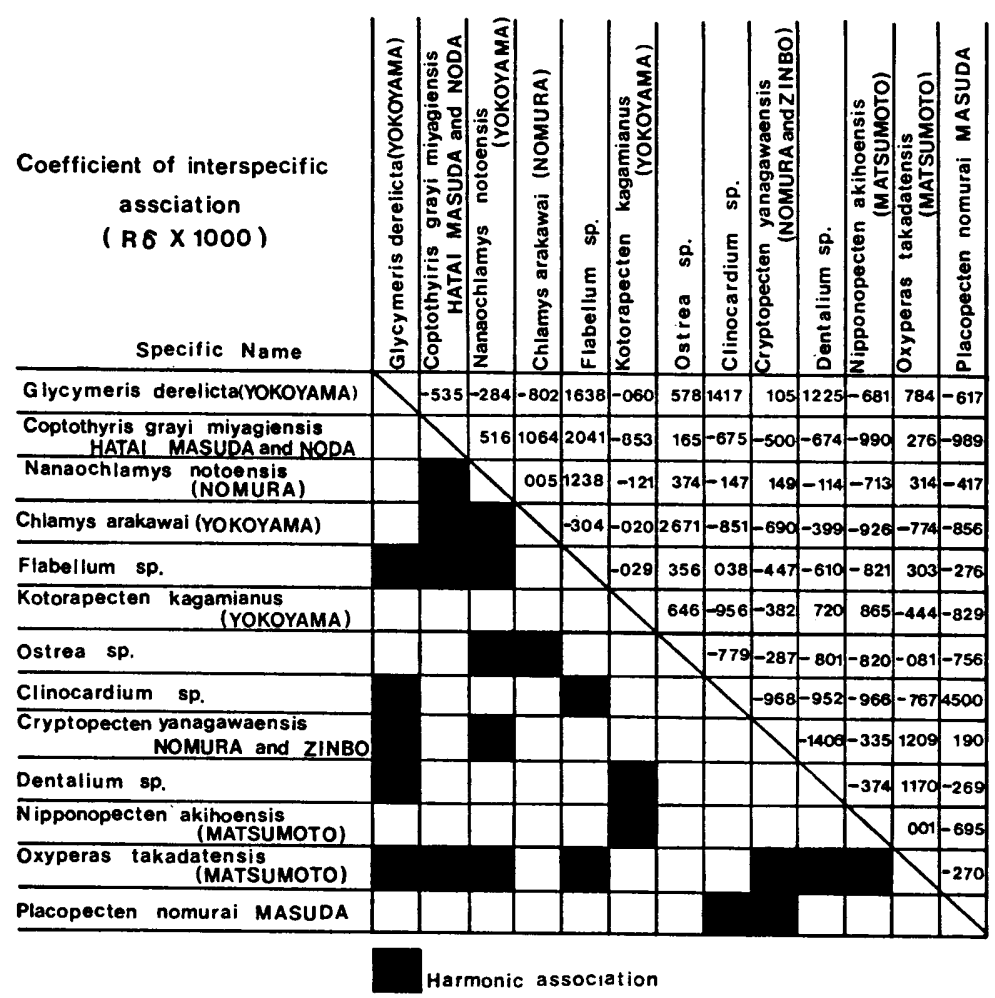

Fig. 5. Coefficient of interspecific associations calculated among characteristic species of the Moniwa Formation (numerical values are shown by a thousand times of $R \delta$ ). 
Affinity:-MASUdA (1958 and 1962) and HAYAMI (1973) considered that $C$. yanagawaensis is an ancestral to $C$. vesiculosus. Subsequently, HAYAMI (1984) mentioned that the phylogeny of Cryptopecten can be divided into $C$. vesiculosus and $C$. nux lines by the manner of disposition of imbricated scales and $C$. vesiculosus is the direct off-shoot of the present species. However, according to the observation of the present author, Cryptopecten from the Kumano Group, Wakayama Prefecture, show an intermediate number of radial ribs between the present species and vesiculosus and its manner of scale disposition is the opposite type. The specimens from the Kumano Group may represent an intermediate taxon between C. yanagawaensis and vesiculosus in that phyletic line.

Associate species:-MoRIsHITA's coefficient of interspecific association (MoRISHITA, 1959), $R \delta$, calculated on the composition matrix of fossil assemblages indicates that present species shows a high degree of association with Glycymris derelicta (Yoкоуама), Nanaochlamys notoensis (YoкоYама), Oxyperas takdatensis (MATsumoto) and Placopecten nomurai MASUda (Fig. 5.). Most of the above species are considered to be ecologically associated with $C$. yanagwaensis, but $N$. notoensis must be regarded to be taphonomically associated because $N$. notoensis is a dweller of rocky and gravelly bottom and is ecologically separated from the others.

Mode of occurrence:-While shells are orientated nearly parallel to the bedding plane with convex side upward at $\mathrm{L} 10$ and they are orientated obliquely or perpendicularly to the bedding plane with convex side downward at $L$ 2, L 9, L 12 and $L$ 18. Shell surfaces, especially radial ribs, ctenolium and ears, are mostly abraded on specimens from most locality except for L 9, L 12 and $\mathrm{L}$ 18. Ctenolium is perfectly preserved at L 9, L 12 and L 18. Attachment scars of sessile animals are found on inner side of shell at $L 2$ and $L$ 10. The above mentioned occurrence suggests that the present species at $\mathrm{L} 9$ may be nearly autochthonous. In accordance with the mode of occurrence, size frequency distribution of shell-height is skewed toward smaller size only at L 9. On account of microfaults fossils are considerably destroyed and deformed at a fossil locality $1 \mathrm{Km}$ north from $\mathrm{L} 2$.

Local variation of distribution in the Moniwa Formation:-Horizontal change of distribution of $C$. yanagawaensis in terms of individuals density (individuals/1 U.) in the Moniwa Formation is shown in Fig. 6. Present species is distributed mainly in eastern area and particularly dense in two parts of that area, one is composed of L 9 and L 10, the type locality of the Moniwa Formation, and another includes $\mathrm{L} 2$ and an additional locality, L 18, which is located $20 \mathrm{Km}$ south from $\mathrm{L} 9$.

Stratigraphical distribution and lithology:-Bihoku Group (Lower sandstone Member):-occurrence; common in medium grained sandstone, Okayama Prefecture. Akeyo Formation (Shukunohora facies) and Oidawara Formation: -occurrence; comon in very coarse grained sandstone and few in granule conglomeratic medium grained sandstone, Gifu Prefecture. Nagaoka Formation:occurrence; few in tuffaceous coarse grained sandstone, Tochigi Prefecture. Sunakozawa Formation:- -ccurrence; common in the tuffaceous sandstone, Kanazawa Prefecture. Yanagawa Formation:-occurrence; few in granule con- 


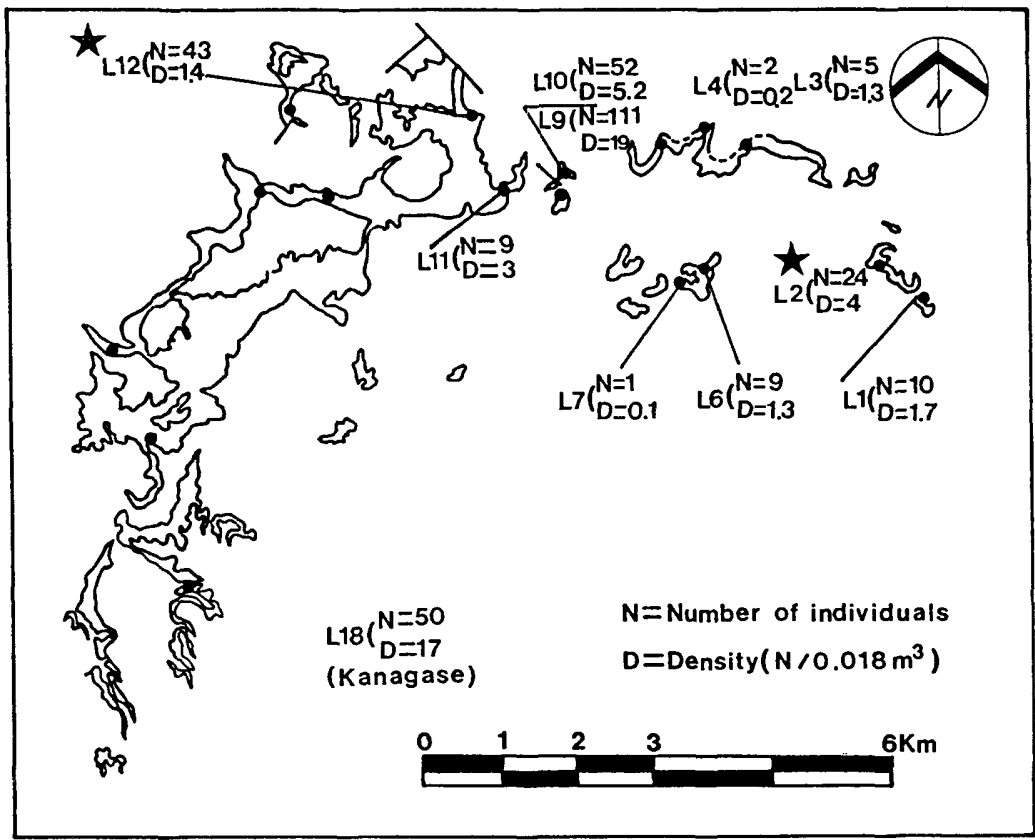

Fig. 6. Local variation of distribution of Cryptopecten yanagawaensis (NomurA and ZiNBo) in the Moniwa Formation. $\mathrm{N}=\mathrm{Number}$ of individuals. $D=$ Density (Number of individuals per $1 \mathrm{Unit}, 0.018 \mathrm{~m}^{3}$. $\star=$ Additionally sampled locality.

glomeratic very coarse grained sandstone, Fukushima Prefecture. Moniwa Formation:-occurrence; common in granule conglomeratic very coarse grained sandstone, Miyagi Prefecture.

Geographical distribution of the present species is limited in the PaleoSetouchi and northern basins of the Early Medial Miocene. Its northern extremity is the Sendai area.

Geological range:-MASUDA (1962 and 1973 a) reported that geological range of the present species is restricted to the Early Miocene. Also in the Paleo-Setouchi province, the Lower sandstone Member of the Bihoku Group is assigned to BLow's number N 8 i.e. the Early Miocene and contemporaneous with the Moniwa Formation. The Oidawara Formation is assigned to BLow's number N 9 i.e. the Medial Miocene (TsuchI et al., 1979 and 1981). Consequently, it seems to be probable that present species ranges in the early Medial Miocene.

Remarks:-Distinct geographic variation of the average number and prominence of radial ribs are not observed. The shells of the Bihoku Group are somewhat thinner than those of the Monirva Formation. Paleogeographical distribution of present species is concordant with inferred paleo-current systems of warm water by CHINzEI (1981). Cryptopecten bullatus and C. vesiculosus living in the Pacific and Japan Sea are warm water elements. They live in shallow sea bottoms consisting of fine sands with pebbles to rocky bottoms free from muddy materials. Lithology o $f$ the fossil localities in the Moniwa Forma- 
tion is harmonious with those substrates of habitats of the living species mentioned above. According to HAYAMI (1984), C. vesiculosus lives in the rather shallower sea than other species. Therefore, it is suggested that there is no ecological differences between the living species and the present species. This assumption is also substantiated by the ecological condition of associate species in the Moniwa. Formation. Present species also occurs in association with Glycymeris cisshuensis and Hyotissa hyotis and is a constituent of Aequipecten Hyotissa Assemblage in the Shukunohora facies of the Mizunami Group (Itogawa, Shibata, Nishimoto and OKumura, 1981). Hayami (1973) pointed out that adherence of serpuloids, bryozoans and many other organisms to the left valves of $C$. vesiculosus is probably owing to the normal living posture with its flatter left valve upside. As noted in earlier lines, convexity of left and right valves is nearly equal in the shells larger than $13 \mathrm{~mm}$ in shell height in the present species. Therefore, it may be considered that the present specis may change its life style corresponding to change in relative convexity of valves (STANLEY, 1970), but further material of C. vesiculosus and present species are necessary to settle this problem. HAYAMI (1973 and 1984) reported that $C$. vesiculosus can be divided into two distinct phenotypes, phenotype $Q$ and $R$, by the value of $100 \mathrm{C} / \mathrm{H}, \mathrm{Q}$ and $\mathrm{R}$, but in the present species two types can not be discriminated (Fig. 7). All the specimens collected from the Moniwa Formation are included into the phenotype $\mathrm{Q}(100 \mathrm{C} / \mathrm{H}=3.85)$.

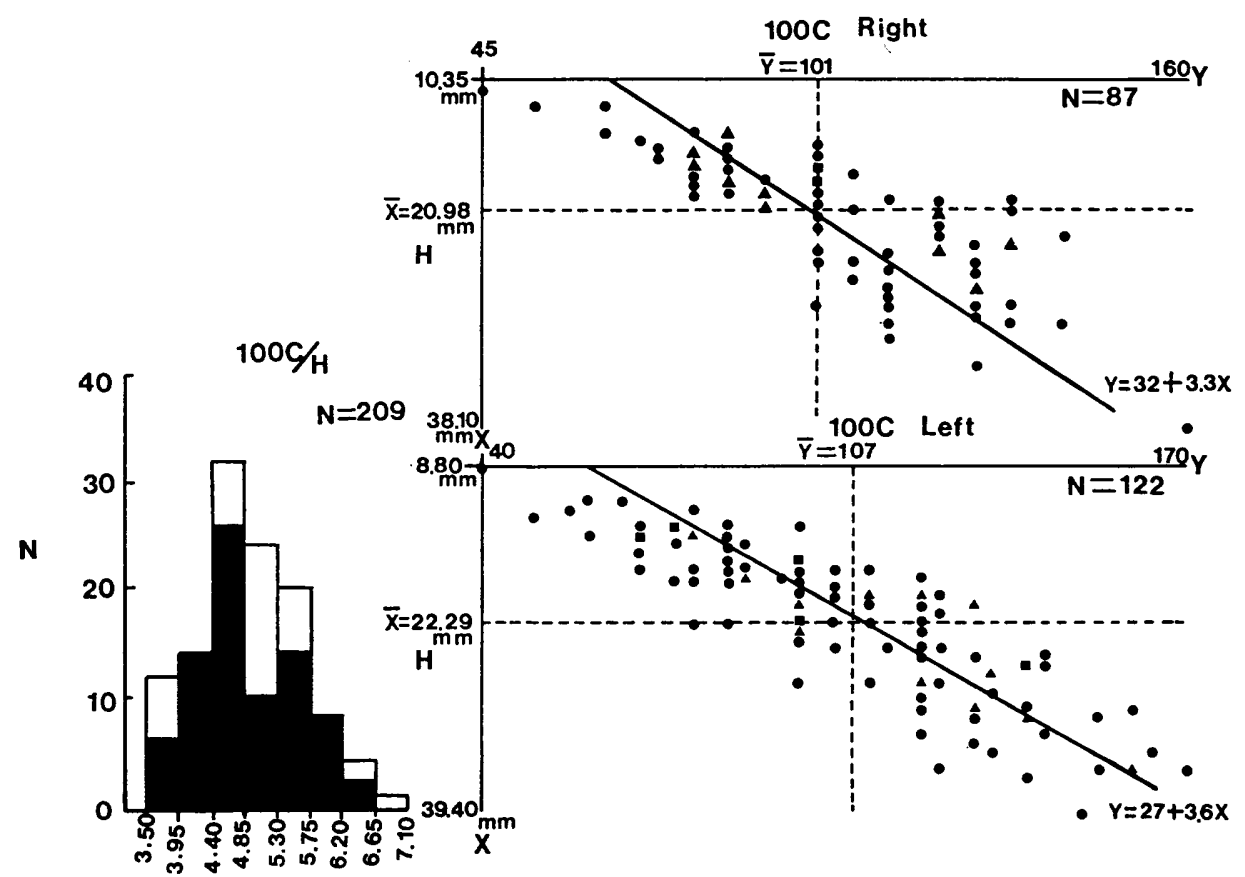

Fig. 7. Scatter diagrams showing the relationship between shell height $(\mathrm{H})$ and prominence of radial ribs (100C) and Frequency distribution of $100 \mathrm{C} / \mathrm{H}$. ( $\bullet=$ one specimen. $\Delta=$ two specimens. $\quad \boldsymbol{\Delta}=$ three specimens.) 


\section{List of Fossil Localities Yielding Present Species}

L 1 (Ezoana): Road-side cliff at the northern slope of a ridge. $100 \mathrm{~m}$ south of a prefectural road from Kagitori to Masuda. Kumanodo, Natori City (Long. $140^{\circ} 51^{\prime} 41^{\prime \prime} \mathrm{E}$, Lat. $38^{\circ} 12^{\prime} 01^{\prime \prime} \mathrm{N}$ ).

L 2 (Jyunijin): Eastern cliff at Jyunijin, $300 \mathrm{~m}$ southwest of a prefectural road from Kagitori to Masuda, Kumanodo, Natori City (Long. $140^{\circ} 50^{\prime} 13^{\prime \prime} \mathrm{E}$, Lat. $\left.38^{\circ} 12^{\prime} 19^{\prime \prime} \mathrm{N}\right)$.

L 3 (Boyo-dai): Southern cliff under Boyo-Dai housing area, Hagurodai, Sendai City (Long. $140^{\circ} 49^{\prime} 05^{\prime \prime} \mathrm{E}$, Lat. $38^{\circ} 13^{\prime} 04^{\prime \prime} \mathrm{N}$ ).

L 4 (Sendai Minami New Town): Road cut at $1.5 \mathrm{Km}$ north from the entrance of a housing area, Moniwa, Sendai City (Long. $140^{\circ} 48^{\prime} 03^{\prime \prime} \mathrm{E}$, Lat. $\left.38^{\circ} 13^{\prime} 05^{\prime \prime} \mathrm{N}\right)$.

L 6 (Osawa): Northern cliff in a swamp area, $300 \mathrm{~m}$ east of $\mathrm{L} 7$, Osawa, Natori City (Long. $140^{\circ} 48^{\prime} 41^{\prime \prime} \mathrm{E}$, Lat. $38^{\circ} 12^{\prime} 12^{\prime \prime} \mathrm{N}$ ).

L 7 (Nakazawa): River-side cliff of Nakazawa, $800 \mathrm{~m}$ upstream from the conjunction with Natori River, Nakazawa, Natori City (Long. $140^{\circ} 48^{\prime} 29^{\prime \prime} \mathrm{E}$, Lat. $38^{\circ} 12^{\prime} 33^{\prime \prime} \mathrm{N}$ ).

L 9 (Type locality of the Moniwa Formation-Lower): River floor at Iwano-sawa under the Moniwa Electric Power Plant, $100 \mathrm{~m}$ upstream from the conjunction with Natori River, Moniwa, Sendai City (Long. $140^{\circ} 47^{\prime} 28^{\prime \prime} \mathrm{E}$, Lat. $38^{\circ} 12^{\prime} 59^{\prime \prime} \mathrm{N}$ ).

L 10 (Type locality of Moniwa Formation-Upper): River-floor of Iwano-sawa $50 \mathrm{~m}$ upstream from L 9, Moniwa, Sendai City. (Long. $140^{\circ} 47^{\prime} 28^{\prime \prime} \mathrm{E}$, Lat $\left.38^{\circ} 12^{\prime} 58^{\prime \prime} \mathrm{N}\right)$.

L 11 (Type locality of the Moniwa Formation-West): Southern cliff in a northwestern small swamp, $6.8 \mathrm{Km}$ west of the Type locality of the Moniwa Formation, Sendai City (Long. $140^{\circ} 47^{\prime} \mathrm{E}$, Lat. $38^{\circ} 12^{\prime} 50^{\prime \prime} \mathrm{N}$ ).

L 12 (Hongo): Road-cut at the Kumanosawa path, $700 \mathrm{~m}$ from the entrance, Moniwa, Sendai City (Long. $140^{\circ} 47^{\prime} \mathrm{E}$, Lat. $38^{\circ} 12^{\prime} 50^{\prime \prime} \mathrm{N}$ ).

L 18 (Kanagase): Road-cut at Shima, $20 \mathrm{~m}$ north of Tohoku New Rail way, Kanagase, Ogawara-machi (Long. $140^{\circ} 41^{\prime} 54^{\prime \prime} \mathrm{E}$, Lat. $38^{\circ} 2^{\prime} 45^{\prime \prime} \mathrm{N}$ ).

MZ (Mizunami): Suganuma, Nakahara Hiyoshi-machi, Mizunami City, Gifu Prefecture (Long. $137^{\circ} 16^{\prime} 41^{\prime \prime} \mathrm{E}$, Lat. $35^{\circ} 24^{\prime} 15^{\prime \prime} \mathrm{N}$ ).

BG (Bihoku Group Lower Horizon): Small cliff along the Kishin railway line at Imai, $300 \mathrm{~m}$ southwest from Tajibe Station, Tajibe, Osa machi, Atetsu-gun, Okayama Prefecture (Long. $133^{\circ} 33^{\prime} 19^{\prime \prime} \mathrm{E}$, Lat. $35^{\circ} 2^{\prime} 27^{\prime \prime} \mathrm{N}$ ).

\section{References Cited}

Chinzer, K. (1981) : Marine biogeography of Japan during Miocene: A reconstraction based on benthonic molluscan faunas (in Japanese). Kaseki (Fossils), (30), 7-14. (1983) : Distribution of the Kadonosawa Fauna (Middle Miocene) and Marine Palaeogeography in Northeast Japan., Origin and migration of Japanese Cenozoic Mollusca (Kotaka, T. and OGASAwA, K. edit.), (in Japanese). 69-76.

Dall, W. E., Bartisch, P. and Rehder, A. H. (1938): A manual of the Recent and fossil marine pelecypod mollusks of the Hawaiian Islands. Bernicece P. Bishop 
Mus. Bull. (153), 1-233, pls. 1-58.

HABE, T. (1977): Systematics of Mollusca in Japan. Bivalvia and Scphopoda (in Japanese). Tokyo, Hokurukan, 1-372, pl. 1-72.

HABE, T. and Kosuge, S. (1967): Shells. Standard Colored Illustration books Vol. 3 (in Japanese). Osaka, Hoikusha. 1-223, pls. 1-64.

Hanzawa, S., Hatai, K., IwaI, J., Kitamura, N. and Shibata, T. (1953) : The Geology of Sendai and its Environs. Sci. Rep. Tohoku Univ. Sendai, [2], (Geol.), 25, 1-50, 2 maps.

Hatai, K., Masuda, K. and Noda, K. (1973) : Marine fossils from the Moniwa Formation, distributed along the Natori River, Sendai, Northeast Honshu, Japan. Part-1, Brachiopoda from the Moniwa Formation, Saito Ho-On Kai Mus., Res. Bull. (42), 9-11, 1 pl.

HAYAMI, I. (1973): Discontinuous variation of evolutionary species, Cryptopecten vesiculosus from Japan. Jour. Paleont., 47, (3), 401-420, pls. 1, 2. (1982): Taxonomic Names of Cryptopecten Species (in Japanese with English abstract). Venus (Japan. Jour. Malac.), 41, (3), 233-236, (short note). (1984): Natural History of Cryptopecten (A Cenozoic Recent Pectinid Groups). Univ. Mus. Univ. Tokyo, Bull., (24), 1-148, pls. 1-13, 1 col. pl.

HAY ^SAKA, S. (1973) : Pliocene Marine Fauna from Tanegashima, South Kyushu, Japan. Sci. Rep. Tohoku Univ. Sendai, [2], (Geol.), Spec. Vol., 6, (Hatai Memorial Volume), 97-108, pls. 6-7.

Firfayama, K. (1954): Miocene Mollusca from the Arakawa Group, Tochigi Prefecture, Japan. (Part-1). Sci. Rep. Tokyo Kyoiku Daigaku, [C], 3, (18), 43-76, pls. $3-5$.

Itoigawa, J., Shibata, H. and Nishimoto, H. (1974) : Molluscan fossils of the Mizunami Group. Bull. Mizunami Fossil Mus., (1), 43-203, pls. 1-63.

Itorgawa, J., Shibata, H., Nishimoto, H. and OKUmURA, Y. (1981) : Miocene fossils of the Mizunami Group, central Japan. 2. Molluscs. Monogr., Mizunami Fossil Mus., (1), 43-203, pls.

Itoigawa, J., Shibata, H., Nishimoto, H. and Okumura, Y. (1982): Miocene fossils of the Mizunami Group, central Japan. 2, Molluscs. Monogr., Mizunami Fossil Mus. (3-B), 1-330.

Kanno, S. (1958): New Tertiary Molluses from the Chichibu Basin, Saitama Prefecture, Central Japan. Sci. Rep. Tokyo Kyoiku Daigaku, [C], 6, (55), 157-229, pls. 1-7.

Masuda, K. (1958) : On the Miocene Pectinidae from the Environs of Sendai. Part-10. On Pecten (Aequipecten) yanagawaensis Nomura and Zinbo. Trans. Proc. Paleont. Soc. Japan, N.S., (30), 189-192.

(1962): Tertiary Pectinidae of Japan. Sci. Rep. Tohoku Univ. Sendai, [2], 33, (2), 117-238, pls. 18-27.

(1966) : Molluscan fauna of the Higashi-Innai Formation of Noto Peninsula Japan. II Remarks on molluscan assemblages and description of species, Trans. Proc. Paleont. Soc. Japan. N.S., (63), 261-293, pls. 35-36.

(1968): Sandpipes penetrating igneous rocks in the environs of Sendai, Ibid., (72), 351-362, pls. 38-39.

(1969) : Molluscan fauna of the Moniwa Formation, Sendai (in Japanese with English abstract). Kaseki (Fossils), (17). 29-40.

(1973a): Molluscan biostratigraphy of the Japanese Neogene (in Japanese with English abstract). Mem. Geol. Soc. Japan, (8), 107-120.

(1973b) : Cenozoic pectinid fossils from Japan. Atlas of Japanese Fossils (in Japanese). Tokyo, Tsukiji Shokan, (33), 1-24, pls. 1-6.

Masuda, K. and Akutsu, J. (1956): A New Fossil Chlamys from the Environs of Utsunomiya City, Japan. Trans. Proc. Paleont. Soc. Japan. N.S., (21), 129-132, pl. 1.

MASUDA, K. and NoDA, H. (1976) : Check list and Bibliography of the Tertiary and 
Quaternary Mollusca of Japan, 1950-1974., Saito Ho-On Kai, Spec. Pub., 1-494. Masuda, K. and TAKegawa, H. (1965): Remarks on the Miocene Mollusca from the Sennan district, Miyagi Prefecture, northeast Honshyu, Japan. Saito Ho-On Kai Mus. Res. Bull., (34), 1-14, pls. 1, 2.

MoRISHITA, M. (1959): Measuring of interspecific association and similarity between communities. Mem. Fac. Sci. Kyushu Univ. [E], (Biol.), 3, (1), 65-80.

NAGAO, T. (1928): Paleogene Fossils of the Islands of Kyushu Japan. Part-2. Sci. Rep. Tohoku Imp. Univ. Sendai, [2], (Geol.), 12, (1), 11-140, pls. 1-17.

Nomura, S. (1940): Molluscan Fauna of the Moniwa Shell Beds Exposed along the Natori-Gawa in the vicinity of Sendai, Miyagi Prefecture. Ibid., 21, (1), 1-46, pls. 1-3.

NomurA, S. and Zinbo, N. (1934): Marine Mollusca from the Ryukyu Limestone of Kikai-Zima Ryukyu Group. Ibid., 16, 109-164, pl. 5.

(1936): Additional Fossil Mollusca from the Yanagawa Shell-Beds in the Fukushima Basin, Northeast Honsyu, Japan. Saito Ho-On Kai Mus., Res. Bull., (10), 335-345, pl. 20.

Nomura, S. and Hatai, K. (1937) : A List of the Miocene Mollusca and Brachiopoda collected from the Region Lying North of the Nanakita River in the Vicinity of Sendai, Rikuzen Province, Japan. Ibid., (13), 121-145, pls. 17-21.

ODA, M. and SAKaI, T. (1977): Microstratigraphy of the Lower to Middle Part of the Hatatate Formation, Sendai, Japan. Fujioka Mem. Vol., 441-456, pls. 1-4.

Ogasawara, K. (1976): Miocene Mollusca from Ishikawa-Toyama Area, Japan. Sci. Rep. Tohoku Univ., [2], (Geol.), 46, (2), 33-78, pl. 11-15.

Oyama, K. (1954): Fossil communities of Oceanic waters (no. 2) (in Japanese with English abstract). Misc. Rep. Res. Inst. Nat Resour., (30), 26-33.

(1973) : Revision of Matajiro Yokoyama's type-Mollusca from the Tertiary and Quaternary of the Kanto Area. Paleont. Soc. Japan, Spec. Papers, (17), 1-148, pls. 1-57.

Sato, Y. (1979) : Geology of the South-Western area of Sendai City and Paleoecology of Molluscan assemblages from Moniwa Formation. 1-92, pls. 1-8. (MS)

(1982): Redescription of Nanaochlamys notoensis (YokOYAMA) and N. notoensis otsutsumiensis (NomURA and HATAI) (Paleontological Study of the Molluscan Fauna from the Moniwa Formation Part-1) Saito Ho-On Kai Mus. Res. Bull., (50), 37-60, pls. $1-3$.

Shikama, T. (1973): Molluscan Assemblages of the basal part of the Zushi Formation in the Miura Peninsula. Sci. Rep. Tohoku Univ. Sendai, [2], (Geol.), Spec. Vol. 6 (Hatai Memorial Volume), 176-204, pls. 16, 17.

Stanley, S. M. (1970) : Relation of shell Form to Life Habits in the Bivalvia. Geol. Soc. America. Mem. 125, 1-496, pl. 1-40.

TAKI, I. and OYAMA, K. (1954): Matajiro Yokoyama's Pliocene and later Faunas from the Kwanto Region in Japan. Palaeont. Soc. Japan. Paleont. Soc. Japan, Spec. Papers, 2, 1-67, pl. 1-49.

Tsuchr, R. et al. (1979): Fundamental Data on Japanese Neogene and Chronology. IGCP-114. Working Group "Japanese Neogene Bio and Chronostratigraphy", $1-156$.

(1981): Ditto. (Supplement). Ibid., 1-124.

Tsuchi, R. (1984): Neogene Biostratigraphy and chronology of Japan. Pacific Neogene Datum Planes, (Contribution to Biostratigraphy and chronology). Edit. by IkeBe, N. and Tsuchi, R. Tokyo University Press., 223-233.

Tsuchi, R. and Shuto, T. (1984): Western Pacific Molluscan Bio-Events and their Relation to Neogene Planktonic Datum Planes. Ibid., 75-81.

VoKes, H. E. (1967) : Genera of the Bivalvia: a systematic and bibliographic catalogue. Bull. Amer. Paleont. 51, (232), 1-394.

(1980): Genera of the Bivalvia: a systematic and bibliographic catalogue., Paleont. Res. Inst. Itahaca, N.Y., 1-307. 
Yokoyama, M. (1911): Pectens from the Koshiba, Neogene. Jour. Geol. Soc. Tokyo, 18, 1-5, pl. 1 .

(1922): Fossils from the Upper Musashino of Kazusa and Shimosa. Jour. Coll. Sci. Imp. Univ. Tokyo, 44, 1-200, pls. 1-17.

\section{Appendix}

Alphabetical index of place names with Japanese writing.
1. Boyo-dai (望洋台)
2. Ezoana (エゾ穴)
3. Goishi (碁石)
4. Hitokita (人来田)
5. Hongo (本郷)
6. Imanari (今成)
7. Jyunijin (十二神)
8. Kanagase (金ケ瀬)
9. Kita-akaishi (北赤石)
10. Mabikizawa (馬引沢)
11. Moniwa (茂庭)
12. Nakazawa (中沢)
13. Nakayachi (中谷地) 14. Osawa (大沢)
15. Sendai Minami New Town (仙台南ニュータウン) 16. Tomioka（富岡） 